\title{
THE HIGHER K-THEORY OF REAL CURVES
}

\author{
CLAUDIO PEDRINI AND CHARLES WEIBEL
}

June 24, 2000

\begin{abstract}
If $X$ is a smooth curve defined over the real numbers $\mathbb{R}$, we show that $K_{n}(X)$ is the sum of a divisible group and a finite elementary abelian 2-group when $n \geq 2$. We determine the torsion subgroup of $K_{n}(X)$, which is a finite sum of copies of $\mathbb{Q} / \mathbb{Z}$ and $\mathbb{Z} / 2$, only depending on the topological invariants of $X(\mathbb{R})$ and $X(\mathbb{C})$, and show that (for $n \geq 2$ ) these torsion subgroups are periodic of order 8 .
\end{abstract}

Let $X$ be a real curve, i.e., a smooth curve $X$ which is defined over $\mathbb{R}$ but not over $\mathbb{C}$. Classical calculations dating to 1883 ([Whd]; see $\S 1$ ) clearly show the relation between the topological invariants of $X(\mathbb{R})$ and the Picard group (and hence $K_{0}$ ) of $X$.

In this paper we shall show that there is a strong connection between the higher algebraic $K$-theory of $X$ and the topological invariants of the underlying space $X(\mathbb{R})$ of its real points, equipped with the Euclidean topology. The link between these will be forged from recent developments in motivic cohomology.

When $X$ is projective, we have the following satisfactory answer.

Main Theorem 0.1. Let $X$ be an irreducible, smooth projective real curve such that $X(\mathbb{R})$ has $\nu>0$ components. Then the groups $K_{n}(X)$ are the direct sum of a divisible group and an elementary abelian 2-group for $n \neq 0$. Moreover, the torsion subgroups $K_{n}(X)_{\text {tors }}$ are periodic of period 8. In fact, if $X$ has genus $g$ then for $n \geq 0$ :

$$
K_{n}(X)_{\text {tors }} \cong \begin{cases}(\mathbb{Q} / \mathbb{Z})^{g} \oplus(\mathbb{Z} / 2)^{\nu-1} & n \equiv 0(\bmod 8) \\ (\mathbb{Z} / 2)^{1+\nu} & n \equiv 1(\bmod 8) \\ (\mathbb{Q} / \mathbb{Z})^{g} \oplus(\mathbb{Z} / 2)^{\nu} & n \equiv 2(\bmod 8) \\ (\mathbb{Q} / \mathbb{Z})^{2} \oplus(\mathbb{Z} / 2)^{\nu-1} & n \equiv 3(\bmod 8) \\ (\mathbb{Q} / \mathbb{Z})^{g} & n \equiv 4(\bmod 8) \\ 0 & n \equiv 5(\bmod 8) \\ (\mathbb{Q} / \mathbb{Z})^{g} & n \equiv 6(\bmod 8) \\ (\mathbb{Q} / \mathbb{Z})^{2} & n \equiv 7(\bmod 8)\end{cases}
$$

Note that $K_{n}(X)$ is divisible for $n \equiv 4,5,6,7(\bmod 8)$.

Pedrini is a member of GNSAGA of CNR, partially supported by MURST and by EC Contract FMR-CT97-0107. Weibel is partially supported by NSF grant DMS98-01560. Both authors would like to thank the Institute for Advanced Study for its hospitality during a crucial part of this project. 
When $X(\mathbb{R}) \neq \emptyset$ and $X$ is affine, the results are similar to the Main Theorem 0.1 and are stated in theorems 4.1 and 7.2. When $X(\mathbb{R})=\emptyset$ the torsion is 4-periodic and is recorded in theorem 6.4.

This paper is organized as follows. After recalling the classical story in $\S 1$, we use Comessatti's theorem to state our transfer argument in $\S 2$. We use this in $\S 3$ to deduce the divisible torsion in $K_{n}(X)$ from the calculations in [PW2]. We use the methods of Merkurjev-Suslin to describe $K_{2}(X)$ in $\S 4$, modulo a minor extension problem which is solved in $\S 6$.

After describing the motivic cohomology of real curves in $\S 5$, we settle the "no loops" case of our Main Theorem in $\S 6$. This includes the case $X(\mathbb{R})=\emptyset$. The Main Theorem 0.1 is proven in $\S 7$.

After this paper was written, Karoubi noticed the similarity between our calculations and the $K R$-theory of $M$, the manifold with involution underlying the complex curve $X_{\mathbb{C}}$. Recall that if $M$ is a space with involution then Atiyah defined an equivariant cohomology theory $K R^{*}(M)$ in [A], using the notion of a Real vector bundle on $M$. If $M$ arises from a real algebraic variety $X$, Atiyah observed that there is a natural map $K_{0}(X) \rightarrow K R^{0}(M)$, and Karoubi $[\mathrm{K}]$ extended it to maps $K_{n}(X) \rightarrow K R^{-n}(M)$ for all $n \geq 0$. Based upon the calculations in this paper, Karoubi and Weibel [KW] have recently shown that for any smooth real variety $X$ the maps $K_{n}\left(X ; \mathbb{Z} / 2^{\nu}\right) \rightarrow$ $K R^{-n}\left(M ; \mathbb{Z} / 2^{\nu}\right)$ are isomorphisms for all $n \geq \operatorname{dim}(X)$.

Notation. By a real variety $X$ we mean a reduced quasi-projective scheme over $\mathbb{R}$ which is not defined over $\mathbb{C}$, i.e., such that the $\mathbb{R}$-algebra $H^{0}\left(X, \mathcal{O}_{X}\right)$ does not contain $\mathbb{C}$. Note that an irreducible real variety is geometrically irreducible. By a real curve we mean a real variety of dimension one.

If $X$ is a real curve, its real locus $X(\mathbb{R})$ is a 1-dimensional topological space; if $X$ is smooth then each component of $X(\mathbb{R})$ is homeomorphic to either an open line or a circle. We write $\nu(X)$ for the number of path components of its real locus $X(\mathbb{R})$. The number of loops, $\lambda(X)$, is the number of circles, i.e., the dimension of $H^{1}(X(\mathbb{R}))$.

We will write $\sigma$ for complex conjugation and $G=\{1, \sigma\}$ for the Galois group of $\mathbb{C} / \mathbb{R}$. Note that if $A$ is a $G$-module, then $\left(A_{\text {tors }}\right)^{G}=\left(A^{G}\right)_{\text {tors }}$, so the notation $A_{\text {tors }}^{G}$ is unambigous.

We shall write $\mathbb{Z} / m(i)$ for the motivic complex of sheaves; by [SV] the restriction of $\mathbb{Z} / m(i)$ to the étale topology is quasi-isomorphic to the sheaf $\mu_{m}^{\otimes i}$. 


\section{$\S 1 . K_{0}$ AND $K_{1}$ OF REAL CURVES}

If $X$ is a connected real curve, it is well known that $K_{0}(X) \cong \mathbb{Z} \oplus \operatorname{Pic}(X)$, where $\operatorname{Pic}(X)$ is the Picard group. In this section we describe how the topological invariants of the underlying space $X(\mathbb{R})$ are reflected in the structure of $\operatorname{Pic}(X)$, and also in the structure of the group $K_{1}(X)$. For convenience, we begin by defining the basic invariants we shall need. We do not assume that $X$ is smooth or proper.

Definitions 1.0. If $X$ is any real curve (not necessarily smooth or proper), let $\nu=\nu(X)$ denote the number of path-connected components of the space $X(\mathbb{R})$, and let $\lambda=\lambda(X)$ denote the number of loops of $X(\mathbb{R})$, i.e., the dimension of $H^{1}(X(\mathbb{R}), \mathbb{Z} / 2)$. Let $E(X)$ denote the number of irreducible algebraic components $X_{i}$ of $X$ which are proper and have no real points i.e., $X_{i}(\mathbb{R})=\emptyset$.

Historically, the first result in this subject is due Weichold and can be found in his 1882 Leipzig thesis [Whd]. We state it here in the form proved later on by Klein (1892) and other authors.

Weichold's Theorem 1.1. Let $X$ be an irreducible, smooth projective real curve, of genus $g$. Then $X(\mathbb{R})$ is the disjoint union of $\nu=\lambda$ circles, and one has

$$
\operatorname{Pic}(X) \cong \mathbb{Z} \times(\mathbb{R} / \mathbb{Z})^{g} \times(\mathbb{Z} / 2)^{\lambda+E-1}
$$

Thus Pic $(X) \otimes \mathbb{Z} / 2 \cong(\mathbb{Z} / 2)^{\lambda}$ if $\nu \neq 0$, while Pic $(X) \otimes \mathbb{Z} / 2 \cong \mathbb{Z} / 2$ if $\nu=0$.

For comparison, recall that $\operatorname{Pic}\left(X_{\mathbb{C}}\right) \cong \mathbb{Z} \times(\mathbb{R} / \mathbb{Z})^{2 g}$. Weichold obtained his result by computing the effect of complex conjugation on the period matrix for the pairing

$$
H_{1}(X(\mathbb{C}), \mathbb{Z}) \times H^{0}\left(X(\mathbb{C}), \Omega_{X}^{1}\right) \rightarrow \mathbb{C}
$$

If we represent an element of $H_{1}$ as a path $\gamma$ in the underlying complex space $X(\mathbb{C})$, then its pairing with a global 1 -form $\omega$ is the path integral $\int_{\gamma} \omega$.

Weichold's theorem has been generalized in several directions. Here is one such result.

Proposition 1.2. [PW1, 1.10] Let $X$ be a real curve. Then

$$
\operatorname{Pic}(X) \otimes \mathbb{Z} / 2 \cong(\mathbb{Z} / 2)^{\lambda(X)+E(X)}
$$

Refinement 1.2.1. If $X$ is an affine curve, $\operatorname{Pic}(X)$ is the direct sum of $(\mathbb{Z} / 2)^{\lambda}$ and a divisible group $D$; this follows from [PW1, 1.2] (or a transfer argument like 2.5 below). However, determining the divisible torsion $D_{\text {tors }}$ in $\operatorname{Pic}(X)$ is a delicate arithmetic question, related to the problem of determining the rank of the group $U(X)$ of units; if $X$ is a connected real curve then $U(X) / \mathbb{R}^{\times}$is a free abelian group.

It turns out that the good invariant is the étale cohomology group $H_{\mathrm{et}}^{1}\left(X, \mu_{\infty}\right)$, which is an extension of $\operatorname{Pic}(X)_{\text {tors }}$ by the divisible group $U(X) \otimes \mathbb{Q} / \mathbb{Z}$. Here $\mu_{\infty}$ is the étale sheaf of all roots of unity on $X$.

A related invariant is the group $H_{\mathrm{et}}^{2}\left(X, \mu_{\infty}\right)$, which is an extension of the Brauer group $\operatorname{Br}(X)$ by $\operatorname{Pic}(X) \otimes \mathbb{Q} / \mathbb{Z}$. It is classical [Witt] $[\mathrm{DK}]$ that $\operatorname{Br}(X)$ is isomorphic to the group $(\mathbb{Z} / 2)^{\nu(X)}$ of maps from the set $\pi_{0} X(\mathbb{R})$ of path components of $X(\mathbb{R})$ to $\mathbb{Z} / 2$. 
If $X$ is an irreducible, smooth projective curve, then $U(X)=\mathbb{R}^{\times}$. By Kummer theory for $\mu_{m} \subset \mathbb{G}_{m}$, we have $H_{\text {et }}^{0}\left(X, \mu_{\infty}\right) \cong \mathbb{Z} / 2$ and the group $H_{\text {et }}^{1}\left(X, \mu_{\infty}\right)$ is isomorphic to $\operatorname{Pic}(X)_{\text {tors }} \cong(\mathbb{Q} / \mathbb{Z})^{g} \oplus(\mathbb{Z} / 2)^{\lambda+E-1}$, the latter isomorphism being part of Weichold's Theorem. The affine case is somewhat different.

Theorem 1.3. Let $X$ be an irreducible, smooth affine real curve, obtained by removing $r$ closed points from a smooth projective curve $\bar{X}$ of genus $g$. If $\nu=\nu(X)$ and $\lambda=\lambda(X)$ then $H_{\mathrm{et}}^{0}\left(X, \mu_{\infty}\right) \cong H_{\mathrm{et}}^{0}\left(\bar{X}, \mu_{\infty}\right) \cong \mathbb{Z} / 2$ and there are natural isomorphisms:

$$
\begin{array}{ll}
H_{\mathrm{et}}^{1}\left(X, \mu_{\infty}\right) \cong(\mathbb{Q} / \mathbb{Z})^{g+r-1} \oplus(\mathbb{Z} / 2)^{\lambda} ; & H_{\mathrm{et}}^{2}\left(X, \mu_{\infty}\right) \cong(\mathbb{Z} / 2)^{\nu} ; \\
H_{\mathrm{et}}^{1}\left(\bar{X}, \mu_{\infty}\right) \cong(\mathbb{Q} / \mathbb{Z})^{g} \oplus(\mathbb{Z} / 2)^{\lambda+E(\bar{X})-1} ; & H_{\mathrm{et}}^{2}\left(\bar{X}, \mu_{\infty}\right) \cong(\mathbb{Q} / \mathbb{Z}) \oplus(\mathbb{Z} / 2)^{\nu} .
\end{array}
$$

Proof. We have already handled $\bar{X}$, so we may suppose that $X$ is an affine real curve. The structure of $\operatorname{Pic}(X)$ implies that $\operatorname{Pic}(X) \otimes \mathbb{Q} / \mathbb{Z}=0$. Hence the group $H_{\text {et }}^{2}\left(X, \mu_{\infty}\right)$ is isomorphic to the Brauer group $\operatorname{Br}(X) \cong(\mathbb{Z} / 2)^{\nu}$. Similarly, because $U(X)$ doesn't contain $\mathbb{C}^{\times}$we have $H_{\text {et }}^{0}\left(X, \mu_{\infty}\right)=\mu_{2} \cong \mathbb{Z} / 2$.

Suppose next that $r=1$, so that $U(X)=\mathbb{R}^{\times}$and $H_{\mathrm{et}}^{1}\left(X, \mu_{\infty}\right) \cong \operatorname{Pic}(X)_{\text {tors }}$. It follows easily from Weichold's Theorem 1.1 and 1.2 that $\operatorname{Pic}(X) \cong(\mathbb{Z} / 2)^{\lambda} \oplus(\mathbb{R} / \mathbb{Z})^{g}$ (see $[\mathrm{PW} 1,1.2])$. The result follows.

If $r>1$, set $X^{\prime}=\bar{X}-\left\{x_{1}\right\}$. Then the Gysin sequence [Mi, p. 244] for $X \subset X^{\prime}$ is

$$
0 \rightarrow H_{\mathrm{et}}^{1}\left(X^{\prime}, \mu_{\infty}\right) \rightarrow H_{\mathrm{et}}^{1}\left(X, \mu_{\infty}\right) \rightarrow(\mathbb{Q} / \mathbb{Z})^{r-1} \stackrel{\tau}{\rightarrow} H_{\mathrm{et}}^{2}\left(X^{\prime}, \mu_{\infty}\right) \rightarrow H_{\mathrm{et}}^{2}\left(X, \mu_{\infty}\right)
$$

Now $H_{\mathrm{et}}^{1}\left(X, \mu_{\infty}\right)$ is the sum of a divisible group and $(\mathbb{Z} / 2)^{\lambda}$ by 1.2 .1 . Thus it suffices to show that the map $\tau$ is zero. Since $X(\mathbb{R})$ is obtained from $X^{\prime}(\mathbb{R})$ by removing points, the map $\pi_{0} X(\mathbb{R}) \rightarrow \pi_{0} X^{\prime}(\mathbb{R})$ is onto. Hence $\operatorname{Br}\left(X^{\prime}\right) \rightarrow \operatorname{Br}(X)$ is an injection, whence $\tau=0$.

Corollary 1.4. Let $X$ be an irreducible, smooth real curve. Then there are natural isomorphisms (with $g, r, \nu, \lambda$ and $E=E(X)$ as in 1.3, and $F$ the function field of $X$ ):

$$
\begin{aligned}
& H_{\mathrm{et}}^{1}(X, \mathbb{Z} / 2) \cong(\mathbb{Z} / 2)^{g+r+\lambda+E} ; \\
& H_{\mathrm{et}}^{2}(X, \mathbb{Z} / 2) \cong(\mathbb{Z} / 2)^{\nu+\lambda+E} ; \\
& H_{\mathrm{et}}^{n}(X, \mathbb{Z} / 2) \cong(\mathbb{Z} / 2)^{\nu+\lambda}, \quad n \geq 3 .
\end{aligned}
$$

Moreover, the image of $H_{\mathrm{et}}^{n}(X, \mathbb{Z} / 2)$ in $H_{\mathrm{et}}^{n}(F, \mathbb{Z} / 2)$ is $(\mathbb{Z} / 2)^{\nu}$ for all $n \geq 2$.

Proof. The $H^{1}$ and $H^{2}$ calculations follow from the Kummer sequence for $\mathbb{Z} / 2 \subset \mu_{\infty}$ and Theorem 1.3, since $H_{\mathrm{et}}^{0}\left(X, \mu_{\infty}\right) \cong \mathbb{Z} / 2$. The description of $H^{n}$ for $n \geq 2$ is given in [PW1, 1.11] or [CT-S, 2.3.1], and is essentially due to Cox [Cox]. (The last assertion is a particular case of a more general result in $[\mathrm{CT}-\mathrm{P}]$.

Variation 1.4.1. Let $N H_{\mathrm{et}}^{n}(X, \mathbb{Z} / 2)$ denote the kernel of $H_{\mathrm{et}}^{n}(X, \mathbb{Z} / 2) \rightarrow H_{\mathrm{et}}^{n}(F, \mathbb{Z} / 2)$, where $F$ is the function field of $X$. That is, $N H_{\mathrm{et}}^{n}(X, \mathbb{Z} / 2)$ is the union over all open $U \subset X$ of the kernels of $H_{\mathrm{et}}^{n}(X, \mathbb{Z} / 2) \rightarrow H_{\mathrm{et}}^{n}(U, \mathbb{Z} / 2)$. 
Since $H_{\mathrm{et}}^{1}(X)$ injects into $H_{\mathrm{et}}^{1}(F), N H_{\mathrm{et}}^{1}(X)=0$. For $n=2$, it is well known that the image of $H_{\text {et }}^{2}(X ; \mathbb{Z} / 2) \rightarrow H_{\text {et }}^{2}(F ; \mathbb{Z} / 2)={ }_{2} \operatorname{Br}(F)$ is the Brauer group $\operatorname{Br}(X) \cong(\mathbb{Z} / 2)^{\nu}$. Hence $N H_{\text {et }}^{2}(X, \mathbb{Z} / 2) \cong \operatorname{Pic}(X) / 2$, a group which is $(\mathbb{Z} / 2)^{\lambda+E}$ by 1.2 .

For $n>2$ we claim that $N H_{\text {et }}^{n}(X, \mathbb{Z} / 2) \cong(\mathbb{Z} / 2)^{\lambda}$. This is clear from the naturality of 1.4 if $\lambda=0$. The case $\lambda(X)>0$ reduces to this because there is an open $U \subset X$ with $\nu(U)=\nu(X)$ but $\lambda(U)=0$.

Lemma 1.5. Let $X$ be a smooth real curve with $X(\mathbb{R}) \neq \emptyset$. Multiplication by $[-1] \in$ $H_{\mathrm{et}}^{1}(\mathbb{R}, \mathbb{Z} / 2)$ induces the map $H_{\mathrm{et}}^{n}(X, \mathbb{Z} / 2) \stackrel{[-1]}{\longrightarrow} H_{\mathrm{et}}^{n+1}(X, \mathbb{Z} / 2)$.

(1) If $n \geq 2$ the map is an isomorphism;

(2) If $n=1$ and $X$ is affine, the map is a surjection;

(3) If $n=1$ and $X$ is projective, the cokernel of the map is $\mathbb{Z} / 2$.

Proof. Write $H^{n}(X)$ for $H_{\mathrm{et}}^{n}(X, \mathbb{Z} / 2)$, and [-1] for the map $H^{n}(X) \rightarrow H^{n+1}(X), x \mapsto$ $x \cup[-1]$. Colliot-Thélène and Scheiderer observed in [CT-S, (2.4)] that the map [-1] fits into a long exact sequence

$$
0 \rightarrow H^{0}(X) \stackrel{\cong}{\rightarrow} H^{0}\left(X_{\mathbb{C}}\right) \stackrel{0}{\rightarrow} H^{0}(X) \stackrel{[-1]}{\longrightarrow} H^{1}(X) \rightarrow H^{1}\left(X_{\mathbb{C}}\right)
$$

$$
\rightarrow H^{1}(X) \stackrel{[-1]}{\longrightarrow} H^{2}(X) \rightarrow H^{2}\left(X_{\mathbb{C}}\right) \rightarrow H^{2}(X) \stackrel{[-1]}{\longrightarrow} H^{3}(X) \rightarrow H^{3}\left(X_{\mathbb{C}}\right) \cdots
$$

If $X$ is affine then $H^{n}\left(X_{\mathbb{C}}\right)=0$ for all $n>1$, and the result follows. If $X$ is projective, this argument works for $n>2$, and shows that $H^{2}(X) \rightarrow H^{3}(X)$ is onto. Since these vector spaces have the same dimension, this map is an isomorphism. Hence the preceeding part of (1.5.1) is exact, namely $H^{1}(X) \rightarrow H^{2}(X) \rightarrow H^{2}\left(X_{\mathbb{C}}\right) \rightarrow 0$. This implies $(3)$ since $H^{2}\left(X_{\mathbb{C}} \cong \mathbb{Z} / 2\right.$.

Remark 1.5.2. Lemma 1.5 may also be easily determined from 1.4, by counting dimensions in (1.5.1). Also, part (3) follows from [CT-S, 2.2.2].

Corollary 1.6. If $E=0$, multiplication by $H_{\mathrm{et}}^{n}(\mathbb{R}, \mathbb{Z} / 2)=\mathbb{Z} / 2$ induces an isomorphism between $\operatorname{Pic}(X) / 2 \cong N H_{\mathrm{et}}^{2}(X, \mathbb{Z} / 2)$ and $N H_{\mathrm{et}}^{n+2}(X, \mathbb{Z} / 2)$ for all $n \geq 0$.

Proof. As the generator of $H_{\mathrm{et}}^{n}(\mathbb{R}, \mathbb{Z} / 2)$ is a power of $[-1]$, this is immediate from 1.5 . 


\section{$\S 2$. Comessatti's Theorem}

In the 1920's, Comessatti used the "pseudonormal" matrix of periods of real integrals to define what we now call the Comessatti characters of the cohomology of a real abelian variety $A$. He computed these characters for the Albanese variety $\operatorname{Alb}\left(X_{\mathbb{C}}\right)$ and its dual, the Picard variety $\operatorname{Pic}^{0}\left(X_{\mathbb{C}}\right)$ of a real variety $X$. In particular his results apply to the Jacobian variety of a real curve. A description of Comessatti's results and their translation into modern language can be found in [CP, 2.5-6].

Here is a modern formulation of Comessatti's key result. We omit its proof, which nowadays is routine; $c f$. [CP, 2.5.3]. For any abelian group $A$ and any integer $i$, it is convenient to write $A(i)$ for the $G$-module which is $A$ with $\sigma$ acting as multiplication by $(-1)^{i}$. We write $A[G]$ for its induced $G$-module, which is the group $A \oplus A$ with $\sigma$ acting as the interchange $\sigma(a, b)=(b, a)$.

Comessatti's Theorem 2.1. Let $\sigma$ be an involution on a free abelian group $H$. Then $H$ decomposes as a module over the group $G=\{1, \sigma\}$ as

$$
H \cong \mathbb{Z}(0)^{a} \oplus \mathbb{Z}(1)^{b} \oplus \mathbb{Z}[G]^{c}, \quad \text { where } \operatorname{dim}(H)=a+b+2 c .
$$

The number $c$ is called the Comessatti character of $H$.

Corollary 2.2. Set $D=H \otimes \mathbb{Q} / \mathbb{Z}$. Then

$$
D^{G}=(\mathbb{Q} / \mathbb{Z})^{a+c} \oplus(\mathbb{Z} / 2)^{b}, \quad H^{1}(G, D) \cong(\mathbb{Z} / 2)^{a} \quad \text { and } \quad H^{2}(G, D) \cong(\mathbb{Z} / 2)^{b} .
$$

In particular, the numbers $a, b$ and $c$ are determined by $D^{G}$ and $\operatorname{dim}(H)$.

Now consider Weichold's case $H=H^{1}(\bar{X}(\mathbb{C}), \mathbb{Z})(1) \cong \mathbb{Z}^{2 g}$, where $\bar{X}$ is projective. There is an equivariant isomorphism $\operatorname{Pic}^{0}\left(\bar{X}_{\mathbb{C}}\right) H \otimes(\mathbb{R} / \mathbb{Z})$, and a routine calculation shows that $\operatorname{Pic}^{0}\left(\bar{X}_{\mathbb{C}}\right)^{G}$ equals $(\mathbb{R} / \mathbb{Z})^{a+c} \oplus(\mathbb{Z} / 2)^{b}$. If $\bar{X}(\mathbb{R}) \neq \emptyset$ then this group also equals $\operatorname{Pic}^{0}(\bar{X})$. Comparing with Weichold's result, we obtain $a+c=g$ and $b$ is $\nu-1$. If $\bar{X}(\mathbb{R})=\emptyset$ then $b$ is 0 when $g$ is even and 1 when $g$ is odd; see [PW1, 1.1.2][CP, 4.1.9] [Sil, 10]. Since $a+b+2 c=2 g$ we can solve this system to get $a=b$ and $c=g-b$.

The calculation for $H=H^{2}(\bar{X}(\mathbb{C}), \mathbb{Z})(1) \cong \mathbb{Z}$ is similar. From the exponential sequence, the group $H \otimes \mathbb{Q} / \mathbb{Z}=H_{\text {et }}^{2}\left(\bar{X}_{\mathbb{C}}, \mu_{\infty}\right)$ is isomorphic to $\operatorname{Pic}\left(\bar{X}_{\mathbb{C}}\right) \otimes \mathbb{Q} / \mathbb{Z} \cong$ $\mathbb{Q} / \mathbb{Z}$. Since $H^{2}\left(\bar{X}, \mu_{\infty}\right) \cong(\mathbb{Q} / \mathbb{Z}) \oplus(\mathbb{Z} / 2)^{\nu}$ by 1.3 , a transfer argument shows that $H_{\mathrm{et}}^{2}\left(\bar{X}_{\mathbb{C}}, \mu_{\infty}\right)^{G}$ contains (and hence equals) $\mathbb{Q} / \mathbb{Z}$. Hence $a=1$ and $b=c=0$. We record these observations:

Corollary 2.3. (Comessatti) Let $\bar{X}$ be an irreducible smooth projective real curve, of genus $g$. Define $b$ to be: $\nu-1$ if $\bar{X}(\mathbb{R}) \neq \emptyset ; 0$ if $\bar{X}(\mathbb{R})=\emptyset$ and $g$ is even; and 1 if $\bar{X}(\mathbb{R})=\emptyset$ and $g$ is odd. Then as a $G$-module we have

$$
\begin{aligned}
\operatorname{Pic}^{0}\left(\bar{X}_{\mathbb{C}}\right) & \cong(\mathbb{R} / \mathbb{Z}(0) \oplus \mathbb{R} / \mathbb{Z}(1))^{b} \oplus(\mathbb{R} / \mathbb{Z})[G]^{g-b} ; \\
H_{\mathrm{et}}^{1}\left(\bar{X}_{\mathbb{C}}, \mu_{\infty}\right) & \cong(\mathbb{Q} / \mathbb{Z}(0) \oplus \mathbb{Q} / \mathbb{Z}(1))^{b} \oplus(\mathbb{Q} / \mathbb{Z})[G]^{g-b} ; \\
H_{\mathrm{et}}^{2}\left(\bar{X}_{\mathbb{C}}, \mu_{\infty}\right) & \cong \mathbb{Q} / \mathbb{Z}(0) .
\end{aligned}
$$

The affine case is almost identical, except that we must distinguish between the $r_{1}$ real points and $r_{2}$ complex points removed from $\bar{X}$ to get $X$, because we must remove $r_{1}+2 r_{2}$ points from $\bar{X}_{\mathbb{C}}$ to get $X_{\mathbb{C}}$. Here we have $H=H^{1}(X(\mathbb{C}), \mathbb{Z})(1) \cong \mathbb{Z}^{2 g+r_{1}+2 r_{2}-1}$ and $H \otimes \mathbb{Q} / \mathbb{Z} \cong H_{\text {et }}^{1}\left(X_{\mathbb{C}}, \mu_{\infty}\right)$. Here is the analogue of 2.3 . 
Corollary 2.4. Let $X$ be an irreducible, smooth affine real curve, which is obtained from a smooth projective curve $\bar{X}$ of genus $g$ by removing $r_{1}$ real points and $r_{2}$ complex points. Let $\nu=\nu(X)$ and $\lambda=\lambda(X)$ denote the number of components and loops of $X(\mathbb{R})$, respectively. Then as an abelian group $H_{\mathrm{et}}^{1}\left(X_{\mathbb{C}}, \mu_{\infty}\right) \cong \mathbb{Q} / \mathbb{Z}^{2 g+r_{1}+2 r_{2}-1}$.

If $X(\mathbb{R}) \neq \emptyset$ then $\nu=r_{1}+\lambda$ and there is a $G$-module isomorphism

$$
H_{\mathrm{et}}^{1}\left(X_{\mathbb{C}}, \mu_{\infty}\right) \cong \mathbb{Q} / \mathbb{Z}(0)^{\nu-1} \oplus \mathbb{Q} / \mathbb{Z}(1)^{\lambda} \oplus(\mathbb{Q} / \mathbb{Z})[G]^{g+r_{2}-\lambda} .
$$

If $X(\mathbb{R})=\emptyset$ then there is a $G$-module isomorphism

$$
H_{\mathrm{et}}^{1}\left(X_{\mathbb{C}}, \mu_{\infty}\right) \cong \mathbb{Q} / \mathbb{Z}(1) \oplus(\mathbb{Q} / \mathbb{Z})[G]^{g+r_{2}-1} .
$$

Note that the exponent of $\mathbb{Q} / \mathbb{Z}(0)$ is $\nu+E(\bar{X})-1$ in both cases of 2.4 .

Proof. If $X(\mathbb{R}) \neq \emptyset$, we have $a+c=g+r_{1}+r_{2}-1$ and $b=\lambda$ by Theorem 1.3 and 2.2. Since $a+b+c=2 g+r_{1}+2 r_{2}-1$, the result follows from 2.1 and the easily checked fact that $\nu=r_{1}+\lambda$. The proof is similar when $X(\mathbb{R})=\emptyset$, except that (as observed in $[\mathrm{PW} 1,1.1 .1])$ we have an extension

$$
0 \rightarrow H_{\mathrm{et}}^{1}\left(X, \mu_{\infty}\right) \rightarrow H_{\mathrm{et}}^{1}\left(X_{\mathbb{C}}, \mu_{\infty}\right)^{G} \rightarrow \mathbb{Z} / 2 \rightarrow 0
$$

Thus Theorem 1.3 yields $H_{\mathrm{et}}^{1}\left(X_{\mathbb{C}}, \mu_{\infty}\right)^{G} \cong(\mathbb{Q} / \mathbb{Z})^{g+r_{2}-1} \oplus \mathbb{Z} / 2$.

We will have frequent recourse to a standard transfer argument, which we formalize like this:

Transfer Argument 2.5. Let $F$ be a functor with transfers, defined on finite field extensions of $\mathbb{R}$. Suppose that $F(\mathbb{C})$ is divisible, and let $\tilde{D}$ denote the maximal divisible subgroup of $F(\mathbb{C})^{G}, G=\operatorname{Gal}(\mathbb{C} / \mathbb{R})$. Then there is an elementary abelian 2-group $E$ and a divisible subgroup $D$ of $F(\mathbb{R})$ such that $D \cong \tilde{D}$ and

$$
F(\mathbb{R}) \cong D \oplus E .
$$

Proof. The hypothesis means that there is a $G$-module structure on $F(\mathbb{C})$, together with maps $\pi^{*}: F(\mathbb{R}) \rightarrow F(\mathbb{C})$ and $\pi_{*}: F(\mathbb{R}) \rightarrow F(\mathbb{C})$ such that $\pi_{*} \pi^{*}$ is multiplication by 2 and $\pi^{*} \pi_{*}=1+\sigma$. Let $D$ denote the subgroup $\pi_{*} F(\mathbb{C})$ of $F(\mathbb{R})$, and set $E=F(\mathbb{R}) / D$. Since $F(\mathbb{C})$ is divisible, so is $D$ and there is a noncanonical decomposition $F(\mathbb{R}) \cong D \oplus E$. Since $D$ contains $2 \cdot F(\mathbb{R}), E$ has exponent 2. Finally, if we set $A=F(\mathbb{C})^{G}$ then the divisible group $\pi^{*} D=(1+\sigma) F(\mathbb{C})$ satisfies $2 \cdot A \subset \pi^{*} D \subset A$; this readily implies that $\pi^{*} D$ is the maximal divisible subgroup of $F(\mathbb{C})^{G}$. Finally, the kernel $H$ of the surjection $\pi^{*}: D \rightarrow \pi^{*} D$ has exponent 2 , so the following standard lemma implies that there is a noncanonical isomorphism $\pi^{*} D \cong D$.

Lemma 2.5.1. If $D$ is a divisible abelian group and $H$ is a subgroup of finite exponent, then the quotient group $D / H$ is (noncanonically) isomorphic to $D$.

Proof. We may assume that $H$ has prime exponent $p$, by induction. Let $I \subseteq D$ be the injective hull of $H$. Then there is a subgroup $D^{\prime}$ so that $D \cong I \oplus D^{\prime}$, and $D / H \cong$ $I / H \oplus D^{\prime}$. So we may assume that $D$ is the injective hull of $H$. In this case, the endomorphism $D \stackrel{p}{\rightarrow} D$ induces the isomorphism $D / H \cong D$. 
Here is an example of the usefulness of the transfer argument. Consider the constant étale sheaf $\mathbb{Q} / \mathbb{Z}$ on a real curve $X$.

Proposition 2.6. Let $X$ be any irreducible, smooth real curve. Let $g, E=E(X)$, $\nu=\nu(X), \lambda=\lambda(X), r_{1}$ and $r_{2}$ be as above. Then:

$$
\begin{gathered}
H_{\mathrm{et}}^{1}(X, \mathbb{Q} / \mathbb{Z}) \cong(\mathbb{Q} / \mathbb{Z})^{g+r_{2}} \oplus(\mathbb{Z} / 2)^{\nu+E}, \\
H_{\mathrm{et}}^{2}(X, \mathbb{Q} / \mathbb{Z}) \cong(\mathbb{Z} / 2)^{\lambda} .
\end{gathered}
$$

Proof. Set $F(k)=H_{\text {et }}^{1}\left(X_{k}, \mathbb{Q} / \mathbb{Z}\right)$. Then $F$ is a functor with transfers defined on fields over $\mathbb{R}$. Now $\sigma$ acts as -1 on $\mu_{\infty}$, as $\sigma(\zeta)=\zeta^{-1}$ for all roots of unity $\zeta$. Hence there is a canonical $G$-module isomorphism $H_{\text {et }}^{1}\left(X_{\mathbb{C}}, \mathbb{Q} / \mathbb{Z}\right) \cong H_{\text {et }}^{1}\left(X_{\mathbb{C}}, \mu_{\infty}\right)(1)$. It follows that $F(\mathbb{C})=H_{\text {et }}^{1}\left(X_{\mathbb{C}}, \mathbb{Q} / \mathbb{Z}\right)$ is divisible. By inspection of 2.3 and 2.4 , we see that in all cases the maximal divisible subgroup of $F(\mathbb{C})^{G}$ is $D=(\mathbb{Q} / \mathbb{Z})^{g+r_{2}}$. The transfer argument 2.5 gives everything except the dimension of the elementary abelian 2-group.

From the Kummer sequence for $\mathbb{Z} / 2 \subset \mathbb{Q} / \mathbb{Z}$ and $H^{0}(X, \mathbb{Q} / \mathbb{Z})=\mathbb{Q} / \mathbb{Z}$, we get from 1.4 that the exponent 2 subgroup of $F(\mathbb{R})$ is

$$
{ }_{2} H_{\mathrm{et}}^{1}(X, \mathbb{Q} / \mathbb{Z}) \cong H_{\mathrm{et}}^{1}(X, \mathbb{Z} / 2) \cong(\mathbb{Z} / 2)^{g+r_{1}+r_{2}+\lambda+E} .
$$

Since $D$ contributes $(\mathbb{Z} / 2)^{g+r_{2}}$ and $\nu=r_{1}+\lambda$, the description of $H_{\text {et }}^{1}$ follows.

Now suppose that $n=2$. If $X$ is projective then $H_{\text {et }}^{2}\left(X_{\mathbb{C}}, \mathbb{Q} / \mathbb{Z}\right) \cong \mathbb{Q} / \mathbb{Z}(1)$ as a $G$-module by 2.3 ; if $X$ is affine then $H_{\text {et }}^{2}\left(X_{\mathbb{C}}, \mathbb{Q} / \mathbb{Z}\right)=0$. In either case, the maximal divisible subgroup of $H_{\text {et }}^{2}\left(X_{C}, \mathbb{Q} / \mathbb{Z}\right)^{G}$ is zero. Hence the transfer argument 2.5 implies that $H_{\text {et }}^{2}(X, \mathbb{Q} / \mathbb{Z})$ is an elementary abelian 2-group. Therefore it is the quotient of $H_{\text {et }}^{2}(X, \mathbb{Z} / 2)=(\mathbb{Z} / 2)^{\nu+\lambda+E}$ by the subgroup $H_{\text {et }}^{1}(X, \mathbb{Q} / \mathbb{Z}) / 2 \cong(\mathbb{Z} / 2)^{\nu+E}$, i.e., $(\mathbb{Z} / 2)^{\lambda}$.

We now describe the étale cohomology of the sheaves $\mu_{\infty}^{\otimes i}=\bigcup_{m} \mu_{m}^{\otimes i}$. Since $\sigma$ sends $\zeta \otimes \cdots \otimes \zeta$ to $\zeta^{-1} \otimes \cdots \otimes \zeta^{-1}$, we see that $\mu_{\infty}^{\otimes i}$ is isomorphic to $\mathbb{Q} / \mathbb{Z}$ for every even $i$, and isomorphic to $\mu_{\infty}$ for every odd $i$.

Proposition 2.7. Let $X$ be any irreducible, smooth real curve. Then for $n \geq 3$ :

$$
H_{\mathrm{et}}^{n}\left(X, \mu_{\infty}^{\otimes i}\right) \cong \begin{cases}(\mathbb{Z} / 2)^{\lambda}, & n+i \text { even } \\ (\mathbb{Z} / 2)^{\nu}, & n+i \text { odd }\end{cases}
$$

Proof. We know that $H_{\mathrm{et}}^{n}\left(X_{\mathbb{C}}, \mu_{\infty}^{\otimes i}\right)=0$, so $H_{\mathrm{et}}^{n}\left(X, \mu_{\infty}^{\otimes i}\right)$ is an elementary abelian 2-group by the usual transfer argument. Again by Kummer theory, $H_{\mathrm{et}}^{n}(X, \mathbb{Z} / 2)=(\mathbb{Z} / 2)^{\nu+\lambda}$ is the sum of $H_{\mathrm{et}}^{n}$ and $H_{\mathrm{et}}^{n-1}$, so the groups may be determined by induction on $n$.

Remark 2.7.1. The calculation for $i$ even, i.e., $H_{\mathrm{et}}^{n}(X, \mathbb{Q} / \mathbb{Z})$, is easily derived from Cox' Theorem [Cox], exactly as in [PW1, 1.8]. See also Scheiderer [Sch, 20.1.6-12].

Remark 2.8. Our transfer argument 2.5 is a simplification of the transfer arguments used in $[\mathrm{PW} 3,5.8]$ and $[\mathrm{CT}-\mathrm{S}, 1.3]$ to calculate to the Chow group $A_{0}(X)$ of (degree zero) zero cycles on a real variety $X$. The reader is invited to compare our argument with the ones in loc. cit. 


\section{§3. Divisible Torsion}

In order to extend the above computations to the groups $K_{n}(X)$ for all $n$ we first recall some known results on the K-theory of complex curves. It is well known that $H^{1}\left(X_{\mathbb{C}}, \mathbb{Q} / \mathbb{Z}\right) \cong(\mathbb{Q} / \mathbb{Z})^{b_{1}}$, where $b_{1}$ is the first Betti number of the topological space $X(\mathbb{C})$. In fact, $b_{1}$ may be read off from Theorem 2.4 .

Theorem 3.1. [PW2, 3.2] Let $X_{\mathbb{C}}$ be a smooth irreducible curve over $\mathbb{C}$. Then $K_{n}\left(X_{\mathbb{C}}\right)$ is divisible for $n \geq 2$, and the torsion subgroup of $K_{n}\left(X_{\mathbb{C}}\right)$ is given by:

$$
K_{n}\left(X_{\mathbb{C}}\right)_{\text {tors }}= \begin{cases}(\mathbb{Q} / \mathbb{Z})^{b_{1}} & \text { if } n \text { is even } \\ \mathbb{Q} / \mathbb{Z} & \text { if } n \text { is odd and } X_{\mathbb{C}} \text { is affine } \\ (\mathbb{Q} / \mathbb{Z})^{2} & \text { if } n \text { is odd and } X_{\mathbb{C}} \text { is projective. }\end{cases}
$$

Equivariant Structure 3.2. The action of $G$ on $K_{n}\left(X_{\mathbb{C}}\right)$ is implicit in the proof of loc. cit., given [PW2, 2.3 and 3.1]. One summand in $K_{2 i-1}\left(X_{\mathbb{C}}\right)_{\text {tors }}$ is the Bott summand $H_{\mathrm{et}}^{0}\left(X, \mu_{\infty}^{\otimes i}\right) \cong \mathbb{Q} / \mathbb{Z}(i)$. When $X$ is projective, the other summand is $H_{\mathrm{et}}^{2}\left(X, \mu_{\infty}^{\otimes i+1}\right) \cong$ $\mathbb{Q} / \mathbb{Z}(i)$, so $K_{2 i-1}\left(X_{\mathbb{C}}\right)_{\text {tors }} \cong \mathbb{Q} / \mathbb{Z}(i)^{2}$. When $n=2 i$, there is a $G$-module isomorphism

$$
K_{2 i}\left(X_{\mathbb{C}}\right)_{t o r s} \cong H_{\mathrm{et}}^{1}\left(X_{\mathbb{C}}, \mu_{\infty}^{\otimes i+1}\right) \cong H_{\mathrm{et}}^{1}\left(X_{\mathbb{C}}, \mu_{\infty}\right)(i)
$$

Theorem 3.3. Let $X$ be a smooth irreducible real curve. Then for all $n \geq 2$ we have a noncanonical isomorphism $K_{n}(X) \cong E_{n} \oplus D_{n}$, where $E_{n}=E_{n}(X)$ is an elementary abelian 2-group and $D_{n}=D_{n}(X)$ is the following divisible group:

$$
\left(D_{n}\right)_{\text {tors }}= \begin{cases}0 & \text { if } n \equiv 1(\bmod 4) ; \\ (\mathbb{Q} / \mathbb{Z})^{g+r_{2}} & \text { if } n \equiv 2(\bmod 4) ; \\ \mathbb{Q} / \mathbb{Z} & \text { if } n \equiv 3(\bmod 4) \text { and } X \text { is affine; } \\ \mathbb{Q} / \mathbb{Z} \oplus \mathbb{Q} / \mathbb{Z} & \text { if } n \equiv 3(\bmod 4) \text { and } X \text { is projective. } \\ (\mathbb{Q} / \mathbb{Z})^{g+r_{1}+r_{2}-1} & \text { if } n \equiv 4(\bmod 4) \text { and } X \text { is affine; } \\ (\mathbb{Q} / \mathbb{Z})^{g} & \text { if } n \equiv 4(\bmod 4) \text { and } X \text { is projective. }\end{cases}
$$

We will see later that $E_{n}(X)$ is finite, and finish the computation of $K_{n}(X)_{\text {tors }}$.

Proof. The functor $F(k)=K_{n}\left(X_{k}\right)$ has transfers, and $F(\mathbb{C})=K_{n}\left(X_{\mathbb{C}}\right)$ is divisible, so the usual transfer argument 2.5 applies: $K_{n}(X)=D_{n} \oplus E_{n}$, where $D_{n}$ is isomorphic to the maximal divisible subgroup of $K_{n}\left(X_{\mathbb{C}}\right)_{\text {tors }}^{G}$.

Suppose first that $n=2 i-1$ is odd. Then the $G$-module $K_{n}\left(X_{\mathbb{C}}\right)_{\text {tors }}$ is either one or two copies of $\mathbb{Q} / \mathbb{Z}(i)$ by 3.2 , depending upon whether $X$ is affine or projective. The result follows as in 2.2 .

Suppose that $n=2 i>0$ and $X$ is projective. We see from 3.2 and 2.3 that

$$
K_{2 i}\left(X_{\mathbb{C}}\right)_{\text {tors }}^{G} \cong \operatorname{Pic}^{0}\left(X_{\mathbb{C}}\right)_{\text {tors }}^{G} \cong(\mathbb{Q} / \mathbb{Z})^{g} \oplus(\mathbb{Z} / 2)^{b},
$$

where $b$ is defined in 2.3. Hence $\left(D_{n}\right)_{\text {tors }} \cong(\mathbb{Q} / \mathbb{Z})^{g}$. 
When $X$ is affine and $X(\mathbb{R}) \neq \emptyset,(3.2 .1)$ and 2.4 yield

$$
K_{2 i}\left(X_{\mathbb{C}}\right)_{\text {tors }}^{G} \cong \begin{cases}(\mathbb{Q} / \mathbb{Z})^{g+r_{1}+r_{2}-1} \oplus(\mathbb{Z} / 2)^{\lambda} & i \text { even; } \\ (\mathbb{Q} / \mathbb{Z})^{g+r_{2}} \oplus(\mathbb{Z} / 2)^{r_{1}+\lambda-1} & i \text { odd }\end{cases}
$$

When $X$ is affine and $X(\mathbb{R})=\emptyset,(3.2 .1)$ and 2.4 yield

$$
K_{2 i}\left(X_{\mathbb{C}}\right)_{\text {tors }}^{G} \cong \begin{cases}(\mathbb{Q} / \mathbb{Z})^{g+r_{2}-1} \oplus(\mathbb{Z} / 2) & i \text { even } \\ (\mathbb{Q} / \mathbb{Z})^{g+r_{2}} & i \text { odd }\end{cases}
$$

In all these cases, we may read off the maximal divisible subgroup, which is isomorphic to $\left(D_{n}\right)_{\text {tors }}$. 


\section{$\S 4 . K_{1} \mathrm{AND} K_{2}$}

In this section we describe $K_{1}(X)$ and $K_{2}(X)$ using off-the-shelf techniques.

First we recall the structure of $K_{1}(X)$ from [PW1]. Classically, we have $K_{1}(X) \cong$ $U(X) \oplus S K_{1}(X)$, where $U(X)$ is the group of global units on $X$. The structure of $U(X)$ is well-known: when $X$ is connected, $U(X)$ is the product of $\mathbb{R}^{\times}$and a finitely generated free abelian group (which vanishes if $X$ is proper). If $X$ is affine with $r$ points at infinity, then the rank of $U(X) / \mathbb{R}^{\times}$is at most $r-1$ by 1.3. Here is the structure of $S K_{1}(X)$ :

Theorem 4.1. ([PW1,5.7]) Let $X$ be a real curve. Then there is a uniquely divisible abelian group $V=V_{1}(X)$ and a natural decomposition:

$$
S K_{1}(X) \cong(\mathbb{Z} / 2)^{\lambda} \oplus V .
$$

We now turn to $K_{2}(X)$. First recall that $K_{2}\left(X_{\mathbb{C}}\right)$ is divisible, and that its torsion subgroup is $H_{\mathrm{et}}^{1}\left(X_{\mathbb{C}}, \mathbb{Q} / Z\right)$. This fact is implicit in $[\mathrm{Su} 2,5.2]$ and explicit in [PW1, 5.6]. The usual transfer argument 2.5 applied to $F(k)=K_{2}\left(X_{k}\right)$, combined with Proposition 2.6, shows that $K_{2}(X)$ is the sum of a divisible group $D_{2}$ with $\left(D_{2}\right)_{\text {tors }} \cong$ $(\mathbb{Q} / \mathbb{Z})^{g+r_{2}}$, and an elementary abelian 2-group.

To say more, we must use the extension

$$
0 \rightarrow H^{1}\left(X, \mathcal{K}_{3}\right) \rightarrow K_{2}(X) \rightarrow H^{0}\left(X, \mathcal{K}_{2}\right) \rightarrow 0
$$

Here $H^{0}\left(X, \mathcal{K}_{2}\right)$ is the image of $K_{2}(X) \rightarrow K_{2}(F)$, where $F$ is the function field $\mathbb{R}(X)$, and the group $H^{1}\left(X, \mathcal{K}_{3}\right)$ is the image of the transfer map $\oplus_{x} K_{2}(x) \rightarrow K_{2}(X)$. Suslin's results in [Su2] yield the following description of the group $H^{0}\left(X, \mathcal{K}_{2}\right)$

Proposition 4.2. Let $X$ be a smooth real curve. Then the Steinberg symbol $\{-1,-1\}$ in $K_{2}(\mathbb{R})$ remains nonzero in $H^{0}\left(X, \mathcal{K}_{2}\right)$, and there is a uniquely divisible group $V_{02}$ such that

$$
H^{0}\left(X, \mathcal{K}_{2}\right) \cong(\mathbb{Q} / \mathbb{Z})^{g+r_{2}} \oplus(\mathbb{Z} / 2)^{\nu+E} \oplus V_{02}
$$

Proof. By $[\mathrm{Su} 2,3.6], H^{0}\left(X, \mathcal{K}_{2}\right)$ contains $K_{2}(\mathbb{R})$. By $[\mathrm{Su} 2,4.4]$ and Theorem 4.1 we have an exact sequence

$$
0 \rightarrow H^{0}\left(X, \mathcal{K}_{2}\right) / 2 \rightarrow H_{\text {et }}^{2}(X, \mathbb{Z} / 2) \rightarrow(\mathbb{Z} / 2)^{\lambda} \rightarrow 0 .
$$

The middle group is $(\mathbb{Z} / 2)^{\nu+\lambda+E}$ by Corollary 1.4 , so the left group is $(\mathbb{Z} / 2)^{\nu+E}$.

We now turn to the other group, $H^{1}\left(X, \mathcal{K}_{3}\right)$. Now $H^{1}\left(X_{\mathbb{C}}, \mathcal{K}_{3}\right)$ is a uniquely divisible group by $[\mathrm{PW} 2,5.5(1)$ and $6.5(3)]$. The usual transfer argument 2.5 shows that $H^{1}\left(X, \mathcal{K}_{3}\right)$ is the sum of a uniquely divisible group $V_{12}$ and an elementary abelian 2-group.

It is convenient to separate out the easy case when $X$ has no real points.

Corollary 4.3. Let $X$ be an irreducible, smooth real curve with $X(\mathbb{R})=\emptyset$. Then there is a uniquely divisible group $V_{2}$ so that:

(1) If $X$ is projective then $K_{2}(X) \cong(\mathbb{Q} / \mathbb{Z})^{g} \oplus(\mathbb{Z} / 2) \oplus V_{2}$, and the $\mathbb{Z} / 2$ summand is generated by $\{-1,-1\}$.

(2) If $X$ is affine, with $r_{2}$ points at infinity, then $K_{2}(X) \cong(\mathbb{Q} / \mathbb{Z})^{g+r_{2}} \oplus V_{2}$. In particular, the symbol $\{-1,-1\}$ is divisible in $K_{2}(X)$. 
Proof. Since $X(\mathbb{R})=\emptyset, K_{2}(x)=K_{2}(\mathbb{C})$ is divisible for every closed point $x$ of $X$. Hence $H^{1}\left(X, \mathcal{K}_{3}\right)$ is divisible, and so uniquely divisible. Therefore $K_{2}(X)_{\text {tors }}$ is $H^{0}\left(X, \mathcal{K}_{2}\right)_{\text {tors }}$, a group which is given by 4.2 , and $K_{2}(X) / 2 \cong(\mathbb{Z} / 2)^{E}$. In particular, if $X$ is affine then $K_{2}(X)$ is divisible.

Twisting 2.4 yields a $G$-module isomorphism $H_{\text {et }}^{1}\left(X_{\mathbb{C}}, \mathbb{Q} / \mathbb{Z}\right) \cong \mathbb{Q} / \mathbb{Z} \oplus(\mathbb{Q} / \mathbb{Z})[G]^{g+r_{2}-1}$; hence $H_{\text {et }}^{1}\left(X_{\mathbb{C}}, \mathbb{Q} / \mathbb{Z}\right)^{G}=(\mathbb{Q} / \mathbb{Z})^{g+r_{2}}$. From $[\operatorname{Su} 2,5.4]$ we see that this is the quotient of $H^{0}\left(X, \mathcal{K}_{2}\right)_{\text {tors }}$ by $K_{2}(\mathbb{R})_{\text {tors }}$. It follows from 4.2 that if $X$ is projective (i.e., $E=1$ ) then $K_{2}(\mathbb{R})$ is a summand of $K_{2}(X)$.

Example 4.3.1 (Brauer-Severi curve). Let $\bar{X}$ be the projective plane curve over $\mathbb{R}$ given by the equation $x^{2}+y^{2}+z^{2}=0$. Note that $\bar{X}(\mathbb{R})=\emptyset$. This is the Brauer-Severi variety corresponding to the quaternion $\mathbb{R}$-algebra $\mathbb{H}$. By Quillen [Q341], $K_{n}(\bar{X}) \cong$ $K_{n}(\mathbb{R}) \oplus K_{n}(\mathbb{H})$. It is well known $[\mathrm{AD}]$ that $K_{2}(\mathbb{H})$ is uniquely divisible, so $K_{2}(\bar{X})_{\text {tors }} \cong$ $(\mathbb{Z} / 2)$. By 4.2 , the nonzero torsion element of $K_{2}(\bar{X})$ is $\{-1,-1\}$.

Removing one (complex) point yields the affine real plane curve $X$ given by the equation $x^{2}+y^{2}=-1$. By 4.3 we see that $K_{2}(X)_{\text {tors }} \cong \mathbb{Q} / \mathbb{Z}$. Here the element $\{-1,-1\}$, which is still nonzero by 4.2 , has become divisible.

If $X(\mathbb{R}) \neq \emptyset$, we need to consider the exact localization sequence

$$
K_{3}(F) \stackrel{\partial}{\rightarrow} \oplus_{x} K_{2}(x) \rightarrow H^{1}\left(X, \mathcal{K}_{3}\right) \rightarrow 0,
$$

or rather its reduction modulo 2. By [MS, 11.11], the image of $\partial$ agrees with the image of the Milnor $K$-group $K_{3}^{M}(F)$. Now Rost and Merkurjev-Suslin have proven that $K_{3}^{M}(F) / 2 \cong H_{\text {et }}^{3}(F, \mathbb{Z} / 2)$ [MS1, 5.1], and we may read this off from 1.4. As the general case is no harder, given Voevodsky's theorem [V], we state our description in that generality.

Reciprocity for Milnor $K$-theory 4.5. Let $F$ be the function field of a smooth real curve $X$. By [Su3], the groups $K_{n}^{M}(F \otimes \mathbb{C})$ are divisible for $n \geq 2$. In fact, they are uniquely divisible for $n \geq 3$; see [PW2, 3.1]. The usual transfer argument shows that $K_{n}^{M}(F)$ is the sum of a uniquely divisible group and the elementary abelian 2-group $K_{n}^{M}(F) / 2$.

Using the norm residue isomorphism $K_{n}^{M}(F) / 2 \cong H_{e t}^{n}(F, \mathbb{Z} / 2)$ and 1.4 , the Gysin sequence for $H_{\mathrm{et}}^{n}(X, \mathbb{Z} / 2)[\mathrm{Mi}$, p. 244] yields the exact sequence for all $n \geq 2$ :

$$
0 \rightarrow(\mathbb{Z} / 2)^{\nu(X)} \rightarrow K_{n}^{M}(F) / 2 \stackrel{\partial}{\rightarrow} \oplus_{x \in X(\mathbb{R})} \mathbb{Z} / 2 \rightarrow(\mathbb{Z} / 2)^{\lambda(X)} \rightarrow 0 .
$$

In particular, if $X=\bar{X}$ is projective, then $\nu(\bar{X})=\lambda(\bar{X})$ and we have the exact sequence

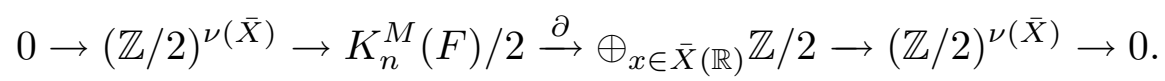

That is, an element of $K_{n}^{M}(F) / 2$ determines a subset of $X(\mathbb{R})$ with an even number points on each compact component, and such a subset determines an element of $K_{n}^{M}(F) / 2$ up to an indeterminacy of $\mathbb{Z} / 2^{\nu}$.

Returning to the exact sequence (4.4), it follows that $H^{1}\left(X, \mathcal{K}_{3}\right) / 2 \cong(\mathbb{Z} / 2)^{\lambda(X)}$. Combining this with the calculation of $H^{0}\left(X, \mathcal{K}_{2}\right)$, we see that we have proven the following result: 
Theorem 4.6. Let $X$ be a smooth real curve with $X(\mathbb{R}) \neq \emptyset$. The group $K_{2}(X)$ is the direct sum of $(\mathbb{Q} / \mathbb{Z})^{g+r_{2}}$, a uniquely divisible group $V_{2}(X)$ and a finite elementary abelian group $E_{2}(X)$. Moreover, there is an exact sequence

$$
0 \rightarrow(\mathbb{Z} / 2)^{\lambda} \rightarrow K_{2}(X)_{\text {tors }} \rightarrow(\mathbb{Q} / \mathbb{Z})^{g+r_{2}} \oplus(\mathbb{Z} / 2)^{\nu} \rightarrow 0
$$

In particular, if $X(\mathbb{R})$ has no loops then $K_{2}(X)_{\text {tors }} \cong(\mathbb{Q} / \mathbb{Z})^{g+r_{2}} \oplus(\mathbb{Z} / 2)^{\nu}$.

Remark 4.6.1. We will solve this extension problem in $\S 7$ below. If $X$ is affine, then we will show that $K_{2}(X)_{\text {tors }} \cong(\mathbb{Q} / \mathbb{Z})^{g+r_{2}} \oplus(\mathbb{Z} / 2)^{\nu}$, and $K_{2}(X) / 2 \cong(\mathbb{Z} / 2)^{\nu}$. However, if $X$ is projective then $K_{2}(X)_{\text {tors }} \cong(\mathbb{Q} / \mathbb{Z})^{g} \oplus(\mathbb{Z} / 2)^{\nu+1}$, and $K_{2}(X) / 2 \cong(\mathbb{Z} / 2)^{\nu+1}$. In this case the sequence of 4.6 splits exactly when $\lambda=1$.

Remark 4.6.2. It is easy to compare $K_{2}(X) / 2$, described in 4.6.1, with the topological $K O$-theory of $X(\mathbb{R})$. From the Atiyah-Hirzebruch spectral sequence we get $K O^{-2} X(\mathbb{R}) \cong(\mathbb{Z} / 2)^{\nu}$, where $X(\mathbb{R})$ has $\nu$ path components.

Example 4.7.1 (projective line). It is well known that the projective line $\mathbb{P}_{\mathbb{R}}^{1}$ has $K_{2}\left(\mathbb{P}_{\mathbb{R}}^{1}\right) \cong K_{2}(\mathbb{R}) \oplus K_{2}(\mathbb{R})$. In this case, $K_{2}\left(\mathbb{P}_{\mathbb{R}}^{1}\right)_{\text {tors }} \cong(\mathbb{Z} / 2)^{2}$ and the extension splits in theorem 4.6.

Example 4.7.2 (the circle). Let $X$ be the affine plane curve over $\mathbb{R}$ given by the familiar equation $x^{2}+y^{2}=1$. Note that $r_{2}=\nu=\lambda=1$ here. It was computed in $[\mathrm{RbW}, 2.1]$ that $K_{2}(X)=K_{2}(\mathbb{R}) \oplus \mathbb{R} / \mathbb{Z}$, and that $H^{1}\left(X, \mathcal{K}_{3}\right)$ is $(\mathbb{Z} / 2)$ on the element $\{-1,-1\} \cdot \eta$, where $\eta$ is the nonzero element of $\operatorname{Pic}(X) \cong \mathbb{Z} / 2$. In this case the extension $0 \rightarrow \mathbb{Z} / 2 \rightarrow K_{2}(X)_{\text {tors }} \rightarrow \mathbb{Q} / \mathbb{Z} \oplus \mathbb{Z} / 2$ in 4.6 does not split.

We conclude this section with a description of $K_{3}(F)$. Since $K_{3}(F \otimes \mathbb{C})$ is divisible by [MS, 11.6], the usual transfer argument 2.5 shows that $K_{3}(F)$ is the sum of a divisible group and an elementary 2-group $E_{3}$. Since $K_{3}(F \otimes \mathbb{C})_{\text {tors }} \cong \mathbb{Q} / \mathbb{Z}$ by $[\mathrm{PW} 2,3.1]$, and $K_{3}(F)$ contains $K_{3}(\mathbb{R})$, we see that the divisible torsion in $K_{3}(F)$ must be $K_{3}(\mathbb{R})_{\text {tors }} \cong$ $\mathbb{Q} / \mathbb{Z}$. It is known that $K_{3}^{M}(F)$ is a subgroup of $K_{3}(F)$, and clearly the torsion subgroup of $K_{3}(\mathbb{R}) \cap K_{3}^{M}(F)$ is $K_{3}^{M}(\mathbb{R})_{\text {tors }} \cong \mathbb{Z} / 2$. By [MS, 11.6], $K_{3}(F)_{\text {tors }}$ is contained in $K_{3}^{M}(F)+K_{3}(\mathbb{R})$. Using reciprocity 4.5 , we have established the following result.

Proposition 4.8. If $F$ is the function field of a smooth real curve $X$, there is an exact sequence:

$$
0 \rightarrow \mathbb{Q} / \mathbb{Z} \oplus(\mathbb{Z} / 2)^{\nu(X)-1} \rightarrow K_{3}(F)_{\text {tors }} \stackrel{\partial}{\rightarrow} \oplus_{x \in X(\mathbb{R})} \mathbb{Z} / 2 \rightarrow(\mathbb{Z} / 2)^{\lambda(X)} \rightarrow 0 .
$$

In particular, $H^{0}\left(X, \mathcal{K}_{3}\right)_{\text {tors }} \cong \mathbb{Q} / \mathbb{Z} \oplus(\mathbb{Z} / 2)^{\nu(X)-1} ;$ the subgroup $\mathbb{Q} / \mathbb{Z}$ is $K_{3}(\mathbb{R})_{\text {tors }}$.

Corollary 4.9. If $X$ is affine, then $K_{3}(X)_{\text {tors }} \cong \mathbb{Q} / \mathbb{Z} \oplus(\mathbb{Z} / 2)^{\nu(X)-1}$. If $X$ is projective then $K_{3}(X)_{\text {tors }} \cong(\mathbb{Q} / \mathbb{Z})^{2} \oplus(\mathbb{Z} / 2)^{\nu(X)-1}$.

Proof. Since both $K_{3}(\mathbb{R})$ and $K_{3}(\mathbb{C})$ are divisible, it follows from the localization sequence that the kernel of the surjection $K_{3}(X) \rightarrow H^{0}\left(X, \mathcal{K}_{3}\right)$ is divisible. Since we know the divisible torsion subgroup of $K_{3}(X)$ from theorem 3.3, the result follows from proposition 4.8 . 


\section{§5. Motivic Cohomology of Real Curves}

The motivic cohomology $H_{M}^{*}(X, \mathbb{Z}(i))$ of $X$ is defined to be its Zariski hypercohomology with coefficients in the complex of sheaves $\mathbb{Z}(i)$, and $H_{M}^{*}(X, \mathbb{Z} / m(i))$ is defined using $\mathbb{Z} / m(i)=\mathbb{Z}(i) \otimes^{\mathbf{L}} \mathbb{Z} / m$ (see $[\mathrm{Fr}, \S 4]$ ). The purpose of this section is to calculate the groups $H_{M}^{*}(X, \mathbb{Z} / m(i))$ when $X$ is a real curve and $m$ is a power of 2 .

Definition 5.1. Let $X$ be a smooth variety over a field $k$ and let $\mu_{m}^{\otimes i}$ be the étale sheaf of $m$-th roots of 1 , twisted $i$ times, where $1 / m \in k$. Write $\mathbf{R} \pi_{*}\left(\mu_{m}^{\otimes i}\right)$ for the total right derived image of the $\mu_{m}^{\otimes i}$ along the change-of-site morphism $\pi: X_{e t} \rightarrow X_{Z a r}$.

We define the complex $B / m(i)$ of Zariski sheaves to be the good truncation of this complex:

$$
B / m(i)=\tau_{\leq i} \mathbb{R} \pi_{*}\left(\mu_{m}^{\otimes i}\right)
$$

Recall that the good truncation $\tau_{\leq i} C$ of a complex $C=C^{*}$ is the natural subcomplex whose cohomology sheaves satisfy:

$$
\mathcal{H}^{q}\left(\tau_{\leq i} C\right)= \begin{cases}\mathcal{H}^{q}(C), & \text { if } q \leq i \\ 0 & \text { if } q>i\end{cases}
$$

In particular, $\mathcal{H}^{q}(B / m(i))$ vanishes if $q>i$, while for $q \leq i$ it is the sheaf $\mathcal{H}^{q}\left(\mu_{m}^{\otimes i}\right)$ associated to the Zariski presheaf $U \mapsto H_{\mathrm{et}}^{q}\left(U, \mu_{m}^{\otimes i}\right)$.

There is a natural map in the derived category of complexes of sheaves in the Zariski topology $[\mathrm{SV}, \S 6][\mathrm{Fr}, \S 6]$ :

$$
\mathbb{Z} / m(i) \rightarrow B / m(i)
$$

Voevodsky [V, 5.5] has proven that (5.2) is an isomorphism when $m$ is a power of 2 . Suslin and Voevodsky (see [SV, 7.2]) have also verified that it is an isomorphism for $i \leq 1$, because $\mathbb{Z}(0) \cong \mathbb{Z}$ and $\mathbb{Z}(1) \cong \mathcal{O}_{X}^{\times}[-1]$, while $\mathbb{Z}(i)=0$ for $i<0$ by definition.

Remark 5.2.1. More generally, it has been conjectured by Beilinson and Lichtenbaum that the map (5.2) is a quasi-isomorphism of complexes of sheaves for all $m$ and $i$. Suslin and Voevodsky [SV , 7.4] have shown that the Beilinson-Lichtenbaum Conjecture holds for those fields $k$ (and $m, i$ ) such that for any field $F$ over $k$ the natural homomorphism

$$
K_{i}^{M}(F) / m \rightarrow H_{e t}^{i}\left(F, \mu_{m}^{\otimes i}\right)
$$

is an isomorphism; this is the so-called Bloch-Kato conjecture in weight $i$ (modulo $m$ ) over $k$. Voevodsky has proved the Beilinson-Lichtenbaum conjecture by proving in $[\mathrm{V}$, $5.1]$ that the Bloch-Kato conjecture holds for all fields $k$ (of characteristic $\neq 2$ ) and all $i$ in the case $m=2^{\nu}$.

The isomorphism (5.2) implies that the Zariski hypercohomology spectral sequence converging to $H_{M}^{p+q}(X, \mathbb{Z} / m(i))$ has:

$$
E_{2}^{p, q}= \begin{cases}H_{\mathrm{Zar}}^{p}\left(X, \mathcal{H}^{q}\left(\mu_{m}^{\otimes i}\right)\right), & \text { if } q \leq i \\ 0 & \text { if } q>i\end{cases}
$$


For purposes of comparison, the Zariski hypercohomology spectral sequence (also known as the local-to-global spectral sequence, or the Bloch-Ogus spectral sequence) is:

$$
\tilde{E}_{2}^{p, q}=H_{\mathrm{Zar}}^{p}\left(X, \mathcal{H}^{q}\left(\mu_{m}^{\otimes i}\right)\right) \Rightarrow H_{e t}^{p+q}\left(X, \mu_{m}^{\otimes i}\right)
$$

Let $N H_{\mathrm{et}}^{n}(X)$ denote the kernel of the map $H_{\mathrm{et}}^{n}(X) \rightarrow H_{\mathrm{et}}^{n}(F)$, where $F$ is the function field of $X$. The group $N H_{\mathrm{et}}^{n}(X)$ is the first level in the coniveau filtration of $H_{\mathrm{et}}^{n}(X)$.

Lemma 5.4. Let $X$ be a smooth variety of dimension d, defined over a field of characteristic zero. Then for all $m=2^{\nu}$ :

$$
H_{M}^{n}(X, \mathbb{Z} / m(i))= \begin{cases}H_{\mathrm{et}}^{n}(X, \mathbb{Z} / m(i)) & \text { if } 0 \leq n \leq i \\ N H_{\mathrm{et}}^{n}(X, \mathbb{Z} / m(i)) & \text { if } n=i+1 \\ H_{\mathrm{Zar}}^{i}\left(X, \mathcal{H}^{d}\left(\mu_{m}^{\otimes i}\right)\right) & \text { if } n=i+d \\ 0 & \text { if } n>i+d\end{cases}
$$

In the missing range $i+1<n<i+d$ there are surjections from $H_{M}^{n}(X, \mathbb{Z} / m(i))$ onto the corresponding level $F i l^{n-i}$ in the coniveau filtration of $H_{\mathrm{et}}^{n}\left(X, \mu_{m}^{\otimes i}\right)$.

Proof. This follows from the fact that there is a morphism of spectral sequences from (5.3) to (5.3.1), which is an isomorphism for $q \leq i$.

Example 5.5. (Suslin $[\mathrm{Su} 3,4.3]$ ) If $X$ is a smooth real variety and $i \geq d$ then:

$$
H_{M}^{n}\left(X_{\mathbb{C}}, \mathbb{Z} / m(i)\right) \cong H_{\mathrm{et}}^{n}\left(X_{\mathbb{C}}, \mu_{m}^{\otimes i}\right) \quad \text { for all } m \text { and } n
$$

Of course this group vanishes unless $n \leq 2 d$. If $m=2^{\nu}$ this follows from 5.4. Indeed, $\mathcal{H}^{q}\left(\mu_{m}^{\otimes i}\right)=0$ for $q>d$, so the spectral sequences (5.3) and (5.3.1) coincide for $i \geq d$.

For example, if $X$ is a real curve then $H_{M}^{1}\left(X_{\mathbb{C}}, \mathbb{Q} / \mathbb{Z}(i)\right) \cong H_{\text {et }}^{1}\left(X_{\mathbb{C}}, \mu_{m}^{\otimes i}\right)$ for $i \geq 1$; this group was determined in 2.3-2.6.

Lemma 5.6. If $X$ is a smooth real curve, then every group $H_{M}^{n}(X, \mathbb{Z} / m(i))$ is finite. This group vanishes if $n \geq i+2$, and equals $H_{\mathrm{et}}^{n}\left(X, \mu_{m}^{\otimes i}\right)$ if $n \leq i$. Finally,

$$
\begin{gathered}
H_{M}^{i+1}(X, \mathbb{Z} / 2(i)) \cong \begin{cases}0 & \text { if } i=0 \\
(\mathbb{Z} / 2)^{\lambda+E} & \text { if } i=1 \\
(\mathbb{Z} / 2)^{\lambda} & \text { if } i \geq 2,\end{cases} \\
H_{M}^{i+1}(X, \mathbb{Q} / \mathbb{Z}(i)) \cong \begin{cases}\mathbb{Q} / \mathbb{Z} & \text { if } i=1 \text { and } X \text { is projective; } \\
0 & \text { if } i \neq 1, \text { or if } i=1 \text { and } X \text { is affine. }\end{cases}
\end{gathered}
$$


Proof. If $m$ is odd then $H_{M}^{n}(X, \mathbb{Z} / m(i))=H_{M}^{n}\left(X_{\mathbb{C}}, \mathbb{Z} / m(i)\right)^{G}$ by the usual transfer argument, so this follows from 5.5. For $m=2^{\nu}$ we may proceed by induction on $\nu$. Thus we may suppose that $m=2$.

Let $F$ be the function field of $X$, and write $H_{\mathrm{et}}^{*}$ for $H_{\mathrm{et}}^{*}(-, \mathbb{Z} / 2)$. By 5.4 we have $H_{M}^{i+1}(X, \mathbb{Z} / 2(i)) \cong N H_{\mathrm{et}}^{i+1}(X)$. We observed in 1.4.1 that $N H_{\mathrm{et}}^{1}(X)=0, N H_{\mathrm{et}}^{2}(X) \cong$ $\operatorname{Pic}(X) / 2 \cong(\mathbb{Z} / 2)^{\lambda+E}$ and $N H_{\text {et }}^{n}(X) \cong(\mathbb{Z} / 2)^{\lambda}$ for $n>2$.

Now consider coefficients $\mathbb{Q} / \mathbb{Z}(i)$. For $i=0$ we need only observe that $H_{\text {et }}^{1}(X, \mathbb{Q} / \mathbb{Z})$ injects into $H_{\text {et }}^{1}(F, \mathbb{Q} / \mathbb{Z})$; this follows from the Gysin sequence, but it could also be obtained from 2.6. For $i=1$ this is the classical fact that $N H_{\text {et }}^{2}\left(X, \mu_{\infty}\right) \cong \operatorname{Pic}(X) \otimes \mathbb{Q} / \mathbb{Z}$ (or one could argue by naturality as in 1.4.1, starting from Theorem 1.3). For $i \geq 2$, we see from 2.7 that $H_{\mathrm{et}}^{i+1}\left(X, \mu_{\infty}^{\otimes i}\right) \cong(\mathbb{Z} / 2)^{\nu(X)}$. As argued in the proof of Theorem 1.3, this injects into $H_{\mathrm{et}}^{i+1}\left(F, \mu_{\infty}^{\otimes i}\right)$, so $H_{M}^{i+1}(X, \mathbb{Z} / 2(i))=0$.

Exercise 5.7. The group $H_{M}^{n}\left(X_{\mathbb{C}}, \mathbb{Z}(i)\right)$ is uniquely divisible for $n \geq 4$ and divisible for $n=3$. If $X$ is affine, it is uniquely divisible for $n=3$ and divisible for $n=2$. These assertions follow from 5.5 using universal coefficients, and are implicit in the proof of [PW2, 1.4].

It follows from the usual transfer argument, 2.7 and 5.6 that when $n \geq 4$ (or $n \geq 3$ if $X$ is affine), $H_{M}^{n}(X, \mathbb{Z}(i))$ is the direct sum of a uniquely divisible group and an elementary abelian 2-group. In fact, since $H_{M}^{n-1}(X, \mathbb{Z}(i))$ is divisible we have $H_{M}^{n}(X, \mathbb{Z}(i))_{\text {tors }} \cong$ $H_{M}^{n-1}(X, \mathbb{Q} / \mathbb{Z}(i))$, and we can read this off from 2.7 using 5.6. If $X$ is affine we have:

$H_{M}^{n}(X, \mathbb{Z}(i))_{\text {tors }} \cong H_{M}^{n-1}(X, \mathbb{Q} / \mathbb{Z}(i)) \cong \begin{cases}(\mathbb{Z} / 2)^{\lambda}, & \text { if } i+1 \geq n \geq 3 \text { and } n+i \text { is even; } \\ (\mathbb{Z} / 2)^{\nu}, & \text { if } i+1 \geq n \geq 3 \text { and } n+i \text { is odd } \\ 0, & \text { otherwise }(n \geq 3) .\end{cases}$

If $X$ is projective and $n=3$, this fails for odd $i$, because $H_{M}^{2}(X, \mathbb{Q} / \mathbb{Z}(i)) \cong \mathbb{Q} / \mathbb{Z} \oplus(\mathbb{Z} / 2)^{\nu}$ by 1.3 . If $i>1$, the summand $\mathbb{Q} / \mathbb{Z}$ survives in $H_{M}^{3}(X, \mathbb{Z}(i))$ because by [PW2, 3.2] it is detected by the group $H_{M}^{3}\left(X_{\mathbb{C}}, \mathbb{Z}(i)\right) \cong \mathbb{Q} / \mathbb{Z}$. However, if $i=1$ then $H_{M}^{3}(X, \mathbb{Z}(1))=0$ and $H_{M}^{2}(X, \mathbb{Z}(1)) \cong H_{\mathrm{Zar}}^{1}\left(X, \mathcal{O}_{X}^{\times}\right) \cong \operatorname{Pic}(X)$. 


\section{$\S 6$. The Spectral Sequence}

Suslin has identified the motivic cohomology groups of a smooth variety with Bloch's Higher Chow groups $\mathrm{CH}^{i}(X, n)$, as defined in [B]. More precisely, the following theorem results from combining [Su3, 2.1] and [FV, 8.2], as in [W, 4.2].

Theorem 6.1. (Suslin) Assume $X$ is a smooth variety over a field $k$ with char $(k)=0$. Then for all $i$ :

$$
C H^{i}(X, n) \cong H_{M}^{2 i-n}(X, \mathbb{Z}(i))
$$

and

$$
C H^{i}(X, n ; \mathbb{Z} / m) \cong H_{M}^{2 i-n}(X, \mathbb{Z} / m(i)) .
$$

Here $C H^{i}(X, n ; \mathbb{Z} / m)$ is the $\bmod \mathbb{Z} / m$ version of the higher Chow groups.

The results in [PW2] were obtained by considering the Bloch-Lichtenbaum spectral sequence $[\mathrm{BL}$ ] converging to the $K$-theory of a field $F$, and the corresponding spectral sequence with finite coefficients (see $[\mathrm{RW}]$ ).

Friedlander and Suslin [FS, 12.13 and 14.2] have recently proven that a BlochLichtenbaum type spectral sequence also exists for every regular $X$. This spectral sequence was also studied by M. Levine in [L]. In addition to the existence of this spectral sequence, we also need some information about its multiplicative properties, which follow from the proof of [FS, 14.2].

Theorem 6.2. (Friedlander-Suslin) Let $X$ be a smooth variety over a field $k$ of characteristic 0 . Then there are spectral sequences converging to $K$-theory and $K$-theory with $\mathbb{Z} / m$ coefficients, defined for $q \leq 0$ :

$$
\begin{gathered}
E_{2}^{p, q}(\mathbb{Z})=H_{M}^{p-q}(X, \mathbb{Z}(-q)) \Rightarrow K_{-p-q}(X ; \mathbb{Z}) ; \\
E_{2}^{p, q}(\mathbb{Z} / m)=H_{M}^{p-q}(X, \mathbb{Z} / m(-q)) \Rightarrow K_{-p-q}(X ; \mathbb{Z} / m)
\end{gathered}
$$

Moreover, there is a multiplicative pairing $E_{2}^{p, q}(\mathbb{Z}) \otimes E_{2}^{p^{\prime}, q^{\prime}}(\mathbb{Z} / m) \rightarrow E_{2}^{p+p^{\prime}, q+q^{\prime}}(\mathbb{Z} / m)$ induced by the product map in motivic cohomology, whose map on abutments is the usual $K$-theory product $K_{m}(X) \otimes K_{n}(X, \mathbb{Z} / m) \rightarrow K_{m+n}(X, \mathbb{Z} / m)$.

Variation. Note that, after reindexing, the Bloch-Lichtenbaum spectral sequence can be written as an Atiyah-Hirzebruch style spectral sequence:

$$
{ }^{\prime} E_{2}^{p, q}=H_{M}^{p}(X, \mathbb{Z} / m(-q / 2)) \Rightarrow K_{-p-q}(X ; \mathbb{Z} / m)
$$

where $\mathbb{Z} / m(-q / 2)=0$ if $q$ is not an even non positive integer.

Remark 6.2.2. Under the "usual" hypotheses for finite coefficients, the proof of [FS, 14.2] shows that the mod $m$ spectral sequence is multiplicative; cf. [L, 8.14]. However, these "usual" hypotheses do not hold for coefficients $\mathbb{Z} / 2$ or even $\mathbb{Z} / 4$ (unless the global functions contain $\sqrt{-1}$ ).

In general, we claim that the spectral sequence $E_{2}^{p q}(\mathbb{Z} / m)$ is multiplicative if either $m$ is odd or if $m=2^{\nu}$ and $\nu \geq 4$. However, we shall not use this result.

To see this claim, recall that the multiplicative structure arises from a map $\rho: \mathbf{M} \rightarrow$ $\mathbf{M} \wedge \mathbf{M}$, where $\mathbf{M}$ is a Moore space, we may cite [O]; see [Wp, 2.5]: If $4 \mid m$ there is a product, compatible under reduction with the product on $H_{M}^{*}(X)$ and $K_{*}(X)$; it is commutative if $8 \mid m$ and associative if $16 \mid m$. 
Proposition 6.3. If $X$ is a smooth real curve, then $K_{n}(X ; \mathbb{Z} / 2)$ is finite for every $n$. In particular the groups $E_{n}(X)=K_{n}(X) / 2$ are finite for every $n$.

Proof. From Theorem 6.2 and Lemmas 5.4 and 5.6 it follows that the group $K_{n}(X ; \mathbb{Z} / 2)$ is a finite group. Since $K_{n}(X) / 2$ is a subgroup of $K_{n}(X ; \mathbb{Z} / 2)$, it is also finite.

Theorem 6.4. Suppose that $X$ is an irreducible, smooth real curve, with $X(\mathbb{R})=\emptyset$. Then $K_{n}(X)$ is a divisible-by-finite group for all $n \geq 2$, and its torsion is 4-fold periodic:

$$
K_{n}(X)_{\text {tors }} \cong \begin{cases}\mathbb{Z} / 2, & n \equiv 1(\bmod 4) ; \\ (\mathbb{Q} / \mathbb{Z})^{g+r_{2}} \oplus(\mathbb{Z} / 2)^{E}, & n \equiv 2(\bmod 4) ; \\ (\mathbb{Q} / \mathbb{Z})^{1+E}, & n \equiv 3(\bmod 4) ; \\ (\mathbb{Q} / \mathbb{Z})^{g+r_{2}+E-1}, & n \equiv 4(\bmod 4) .\end{cases}
$$

Proof. By 3.3 we have $K_{n+1}(X) \otimes \mathbb{Q} / \mathbb{Z}=0$, so $K_{n+1}(X, \mathbb{Q} / \mathbb{Z}) \cong K_{n}(X)_{\text {tors }}$. Because $X(\mathbb{R})=\emptyset$, there are no differentials in the spectral sequence 6.2 , so we have

$$
K_{n}(X, \mathbb{Q} / \mathbb{Z}) \cong \begin{cases}H^{0}(X, \mathbb{Q} / \mathbb{Z}(i)) \oplus H^{2}(X, \mathbb{Q} / \mathbb{Z}(i+1)), & n=2 i \\ H^{1}(X, \mathbb{Q} / \mathbb{Z}(i)), & n=2 i-1 .\end{cases}
$$

The result follows by plugging in the values of these groups from 1.3, 2.6 and 2.7.

Example 6.5 (Brauer-Severi curve). Let $\bar{X}$ be the projective plane curve over $\mathbb{R}$ given by the equation $x^{2}+y^{2}+z^{2}=0$, i.e., the Brauer-Severi variety corresponding to the quaternion $\mathbb{R}$-algebra $\mathbb{H}$. By Quillen [Q341], $K_{n}(\bar{X}) \cong K_{n}(\mathbb{R}) \oplus K_{n}(\mathbb{H})$.

The group structure of $K_{*}(\mathbb{H}, \mathbb{Z} / m)$ was determined by Suslin [Su1, 3.5]; it is isomorphic with finite coefficients to the homotopy groups of $\mathbb{Z} \times B S p \simeq \Omega^{4} B O$ because the symplectic group $S p$ is homotopy equivalent to $B G L\left(\mathbb{H}^{t o p}\right)$. In particular, we have $K_{n}(\mathbb{H})_{\text {tors }} \cong K_{n+4}(\mathbb{R})_{\text {tors }}$ for all $n \geq 0$.

It is an amusing exercise to check that our Proposition 6.4 agrees with the QuillenSuslin calculation for $\bar{X}$.

Now suppose that $X(\mathbb{R}) \neq \emptyset$, i.e., $\nu>0$, and pick $\nu$ real points of $X$, one on each component of $X(\mathbb{R})$. The inclusion of these points in $X$ induces a map from $H^{n}(X, \mathbb{Z} / 2)$ to $\oplus_{i=1}^{\nu} H_{\mathrm{et}}^{n}(\mathbb{R}, \mathbb{Z} / 2) \cong(\mathbb{Z} / 2)^{\nu}$.

Lemma 6.6. The map $H^{n}(X, \mathbb{Z} / 2) \rightarrow(\mathbb{Z} / 2)^{\nu}$ is onto for all $n \geq 1$.

Proof. Since $X$ is smooth affine, the maps $H_{\mathrm{et}}^{n}(X, \mathbb{Z} / 2) \rightarrow H_{\mathrm{et}}^{n+1}(X, \mathbb{Z} / 2)$ are onto by lemma 1.5. Since $H_{\mathrm{et}}^{n}(\mathbb{R}, \mathbb{Z} / 2) \rightarrow H_{\mathrm{et}}^{n+1}(\mathbb{R}, \mathbb{Z} / 2)$ are isomorphisms, we may assume that $n>2$. The result now follows from the natural isomorphisms $H_{\mathrm{et}}^{n}(X, \mathbb{Z} / 2) \cong$ $\oplus H^{i}(X(\mathbb{R}), \mathbb{Z} / 2), n>2 \operatorname{dim}(X)$ in Cox' Theorem [Cox], formalized in [CT-S, 2.3.1]. Indeed, the following commutative diagram is a special case:

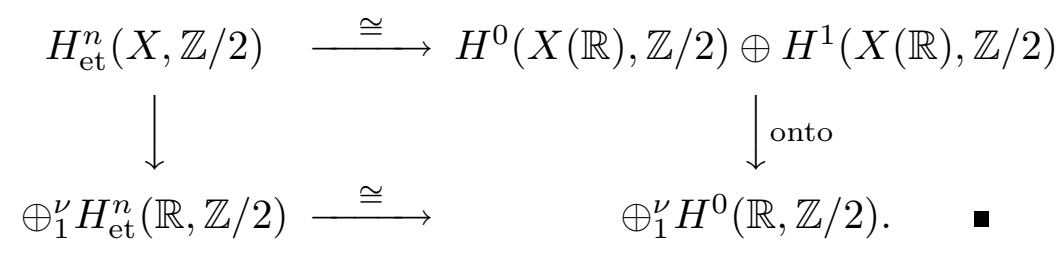


The choice of these $\nu$ points yields a morphism of spectral sequences (6.2), from the one converging to $K_{*}(X, \mathbb{Z} / 2)$ to the sum of $\nu$ copies of the one converging to $K_{*}(\mathbb{R}, \mathbb{Z} / 2)$. By 6.6, the morphism has a surjection in the $(p, q)$ spot for all $p>q, p \leq 0$.

We now turn to real curves such that $X(\mathbb{R})$ has no loops, and $X(\mathbb{R}) \neq \emptyset$. If $n \geq 2$ then $H^{n}(X, \mathbb{Z} / 2) \cong(\mathbb{Z} / 2)^{\nu}$ by 1.4 , so the $\operatorname{map} H^{n}(X, \mathbb{Z} / 2) \rightarrow(\mathbb{Z} / 2)^{\nu}$ of 6.6 is an isomorphism. That is, the morphism of spectral sequences has an isomorphism in the $(p, q)$ spot for all $p \geq q+2$.

By [RW, 5.3] the classes in bidegrees $(p, q)$ with $p=-4 k-2$ or $p=-4 k-3$ support nontrivial differentials in the spectral sequence for $\mathbb{R}$. It follows that when $p \equiv-3$ and $p \equiv-2$ and $p>q$ the differentials $E_{2}^{p q} \rightarrow E_{2}^{p+2, q-1}$ are onto in the spectral sequence for $X$. When $p \equiv-3$ and $p \equiv-2$ and $p=q$ the differential (for $X$ ) goes from $E_{2}^{p p} \cong \mathbb{Z} / 2$ to $E_{2}^{p+2, p-1} \cong(\mathbb{Z} / 2)^{\nu}$ and must be injective by the same argument. When $p \equiv-1$ and $p=q$ the differential is zero; this follows by the same argument because by [RW, 5.3] the corresponding differential in the spectral sequence for $\mathbb{R}$ vanishes.

If we write $\tilde{H}^{1}$ for the kernel of the surjection $H_{\text {et }}^{1}(X, \mathbb{Z} / 2) \rightarrow(\mathbb{Z} / 2)^{\nu}$, then the first few columns of the spectral sequence look like this:

$\begin{array}{ccccc}\text {-3 } & \text {-2 } & \frac{-1}{n} & \frac{p=0}{\mathbb{Z} / 2} & \frac{+1}{0} \\ & & \mathbb{Z} / 2 & H^{1} & \operatorname{Pic}(X) / 2 \\ & \mathbb{Z} / 2 & H^{1} & \nu & 0 \\ \mathbb{Z} / 2 & H^{1} & \nu & \nu & 0 \\ H^{1} & \nu & \nu & \nu & 0 \\ \nu & \nu & \nu & \nu & 0 \\ \nu & \nu & \nu & \nu & 0\end{array}$

$E_{2}$ terms for $K_{*}(X, \mathbb{Z} / 2)$

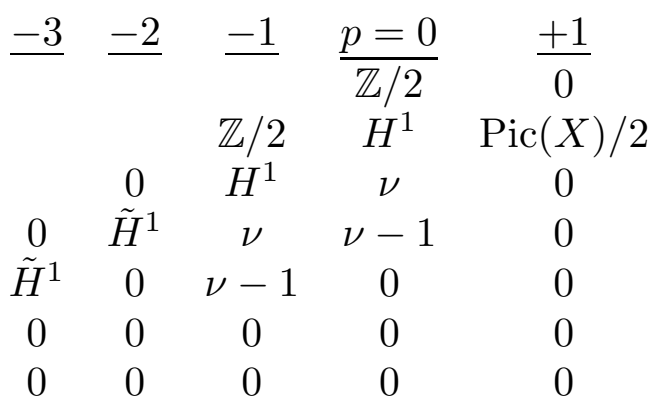

$E_{3}$ terms for $K_{*}(X, \mathbb{Z} / 2)$

Here the symbol " $\nu$ " represents $(\mathbb{Z} / 2)^{\nu}$, and similarly with " $\nu-1$ ". Every set of four columns to the left is the same as the set from $p=0$ to $p=-3$, shifted left 4 and down 4. By inspection, there are no further differentials, and $E_{3}=E_{\infty}$. Thus the associated graded groups for the filtration of $K_{*}(X, \mathbb{Z} / 2)$ may be read off from (6.7).

Warning 6.7.1. There are some nontrivial extensions in the filtration of $K_{*}(X, \mathbb{Z} / 2)$ coming from (6.7). For example, $K_{8 k+2}(\mathbb{R}, \mathbb{Z} / 2) \cong \mathbb{Z} / 4$ is a subgroup of $K_{8 k+2}(X, \mathbb{Z} / 2)$, which must equal $\mathbb{Z} / 4 \oplus(\mathbb{Z} / 2)^{\nu-1}$. Thus the extensions

$$
0 \rightarrow(\mathbb{Z} / 2)^{\nu} \rightarrow K_{8 k+2}(X, \mathbb{Z} / 2) \rightarrow \mathbb{Z} / 2 \rightarrow 0
$$

do not split. Similarly, it can be shown that $K_{8 k+3}(X, \mathbb{Z} / 2)$ contains $\nu-1$ copies of $\mathbb{Z} / 4$. We omit the argument as we do not need this fact. 
Theorem 6.8. Let $X$ be a real curve such that $X(\mathbb{R})$ has $\nu>0$ components and no loops. Then the groups $K_{n}(X)_{\text {tors }}$ are periodic of period 8 for $n>0$. In fact:

$$
K_{n}(X)_{\text {tors }} \cong \begin{cases}\text { Pic }(X)_{\text {tors }} & n=0 \\ \mathbb{Z} / 2 & n \equiv 1(\bmod 8) \\ (\mathbb{Q} / \mathbb{Z})^{g+r_{2}} \oplus(\mathbb{Z} / 2)^{\nu} & n \equiv 2(\bmod 8) \\ \mathbb{Q} / \mathbb{Z} \oplus(\mathbb{Z} / 2)^{\nu-1} & n \equiv 3(\bmod 8) \\ (\mathbb{Q} / \mathbb{Z})^{g+r_{2}+\nu-1} & n \equiv 4(\bmod 8) \\ 0 & n \equiv 5(\bmod 8) \\ (\mathbb{Q} / \mathbb{Z})^{g+r_{2}} & n \equiv 6(\bmod 8) \\ \mathbb{Q} / \mathbb{Z} & n \equiv 7(\bmod 8) \\ (\mathbb{Q} / \mathbb{Z})^{g+r_{2}+\nu-1} & n \equiv 0(\bmod 8), n \geq 8\end{cases}
$$

Note that $K_{n}(X)$ is divisible for $n \geq 2$ unless $n \equiv 1,2,3(\bmod 8)$.

Before beginning the proof, we make some preliminary observations. Because there are no loops, $X$ is affine and $r_{1}=\nu$ (see 2.4). By 1.4, $\operatorname{dim} H_{\mathrm{et}}^{1}(X, \mathbb{Z} / 2)=g+r_{2}+\nu$ and $\operatorname{dim} \tilde{H}^{1}=g+r_{2}$. The groups $\left(D_{n-1}\right)_{\text {tors }} \cong(\mathbb{Q} / \mathbb{Z})^{a}$ given in Theorem 3.3 must contribute $(\mathbb{Z} / 2)^{a}$ to $K_{n}(X, \mathbb{Z} / 2)$ by the universal coefficient theorem. For $n \equiv 1, \ldots, 4$ the numbers $a$ are $0, g+r_{2}, 1$ and $g+r_{2}+\nu-1$, respectively.

Proof. It is easiest to begin at $K_{6}(X, \mathbb{Z} / 2)$; it is 0 by (6.7). This implies that $K_{6}(X)$ is divisible and that $K_{5}(X)$ is torsionfree. By 3.3, we have $K_{6}(X)=D_{6}$ and $K_{5}(X)=D_{5}$. Hence $K_{6}(X)_{\text {tors }}=\left(D_{6}\right)_{\text {tors }}=(\mathbb{Q} / \mathbb{Z})^{g+r_{2}}$, and $K_{5}(X)$ is uniquely divisible.

Working backwards from $K_{5}$, this implies that the exponent 2 subgroup of $K_{4}(X)$ is $K_{5}(X, \mathbb{Z} / 2)$, which by $(6.7)$ is $\tilde{H}^{1} \oplus(\mathbb{Z} / 2)^{\nu-1}$. But $D_{4}$ accounts for all of this torsion, whence $K_{4}(X)=D_{4}(X)$. This implies that the exponent 2 subgroup of $K_{3}(X)$ is $K_{4}(X, \mathbb{Z} / 2)=(\mathbb{Z} / 2)^{\nu} ;$ since $K_{3}(X)$ contains $K_{3}(\mathbb{R})_{\text {tors }} \cong \mathbb{Q} / \mathbb{Z}$, we see that $K_{3}(X)_{\text {tors }}$ is $\mathbb{Q} / \mathbb{Z} \oplus(\mathbb{Z} / 2)^{\nu-1}$.

Working forwards from $K_{6}, K_{6}(X)_{\text {tors }}$ accounts for all of $K_{7}(X, \mathbb{Z} / 2) \cong(\mathbb{Z} / 2)^{g+r_{2}}$, so $K_{7}(X)$ is divisible and $K_{7}(X)_{\text {tors }}=\left(D_{7}\right)_{\text {tors }} \cong \mathbb{Q} / \mathbb{Z}$. This in turn accounts for $K_{8}(X, \mathbb{Z} / 2) \cong \mathbb{Z} / 2$, so $K_{8}(X)$ is divisible. In turn, $K_{8}(X)_{\text {tors }} \cong\left(D_{8}\right)_{\text {tors }}$ accounts for $g+r_{2}+\nu-1$ terms in $K_{9}(X, \mathbb{Z} / 2) \cong H^{1}$. Therefore $K_{9}(X)_{\text {tors }}=K_{9}(X) / 2 \cong \mathbb{Z} / 2$.

We now turn to $K_{10}(X, \mathbb{Z} / 2)$. Since $K_{10}(\mathbb{R}, \mathbb{Z} / 2) \cong \mathbb{Z} / 4$ is a summand, we must have $K_{10}(X, \mathbb{Z} / 2) \cong \mathbb{Z} / 4 \oplus(\mathbb{Z} / 2)^{\nu-1}$. Since $K_{9}(X)_{\text {tors }} \cong \mathbb{Z} / 2$, it follows that $K_{10}(X) / 2 \cong$ $(\mathbb{Z} / 2)^{\nu}$. Hence $K_{10}(X)_{\text {tors }}$ is the sum of $(\mathbb{Z} / 2)^{\nu}$ and $\left(D_{10}\right)_{\text {tors }} \cong(\mathbb{Q} / \mathbb{Z})^{g+r_{2}}$.

Since the columns in the spectral sequence are 8 -fold periodic, the same argument works for all other values of $n \geq 3$. One could argue similarly for $K_{1}$ and $K_{2}$, but we prefer to cite 4.1 and 4.6.

Folklore Calculation 6.9. If $X$ is obtained from the affine line $\operatorname{Spec}(\mathbb{R}[t])$ by removing $\nu-1$ real points and $r_{2}$ complex points, then the localization sequence for $X \subset \operatorname{Spec}(\mathbb{R}[t])$ yields a decomposition:

$$
K_{n}(X) \cong K_{n}(\mathbb{R}) \oplus K_{n-1}(\mathbb{R})^{\nu-1} \oplus K_{n-1}(\mathbb{C})^{r_{2}} .
$$

This result agrees with our formulas for the torsion, as one can easily check. 


\section{§7. ElEmEntary 2-TORSION FOR CURVES}

In this section we assemble the information into a calculation of the 2-torsion in $K_{*}(X)$ when $\lambda \neq 0$. The following trick allows us to adopt the simplifying assumption that $X$ is affine, since it implies the projective calcultion.

Lemma 7.1. Let $\bar{X}$ be an irreducible, smooth projective real curve with $\bar{X}(\mathbb{R}) \neq \emptyset$. If $p$ is any real point and $X=\bar{X}-\{p\}$ then for all $n$ :

$$
K_{n}(\bar{X}) \cong K_{n}(X) \oplus K_{n}(\mathbb{R})
$$

Proof. Let $\pi: \bar{X} \rightarrow \operatorname{Spec}(\mathbb{R})$ denote the structure map and $\iota: \operatorname{Spec}(\mathbb{R})=p \rightarrow \bar{X}$ the inclusion. Then the composition $\pi \iota$ is the identity, and both maps are proper. Hence the $\operatorname{map} \iota_{*}: K_{n}(\mathbb{R}) \rightarrow K_{n}(\bar{X})$ is an injection, split by $\pi_{*}$ for all $n$. Therefore the localization sequence breaks up into split exact sequences $0 \rightarrow K_{n}(\mathbb{R}) \stackrel{\iota_{*}}{\longrightarrow} K_{n}(\bar{X}) \rightarrow K_{n}(X) \rightarrow 0$.

Theorem 7.2. Let $X$ be an affine real curve such that $X(\mathbb{R})$ has $\nu>0$ components and $\lambda$ loops. Then the groups $K_{n}(X)_{\text {tors }}$ are periodic of period 8 for $n>0$. In fact:

$$
K_{n}(X)_{\text {tors }} \cong \begin{cases}\operatorname{Pic}(X)_{\text {tors }} & n=0 \\ (\mathbb{Z} / 2)^{1+\lambda} & n \equiv 1(\bmod 8) \\ (\mathbb{Q} / \mathbb{Z})^{g+r_{2}} \oplus(\mathbb{Z} / 2)^{\nu} & n \equiv 2(\bmod 8) \\ (\mathbb{Q} / \mathbb{Z}) \oplus(\mathbb{Z} / 2)^{\nu-1} & n \equiv 3(\bmod 8) \\ (\mathbb{Q} / \mathbb{Z})^{g+r_{1}+r_{2}-1} & n \equiv 4(\bmod 8) \\ 0 & n \equiv 5(\bmod 8) \\ (\mathbb{Q} / \mathbb{Z})^{g+r_{2}} & n \equiv 6(\bmod 8) \\ (\mathbb{Q} / \mathbb{Z})^{g+r_{1}+r_{2}-1} \oplus(\mathbb{Z} / 2)^{\lambda} & n \equiv 0(\bmod 8), n \geq 8 \\ (\mathbb{Q} / \mathbb{Z})^{g+(\bmod 8)}\end{cases}
$$

Note that $K_{n}(X)$ is divisible for $n \equiv 4,5,6,7(\bmod 8)$.

Proof of Main Theorem 0.1. Let $\bar{X}$ be an irreducible, smooth projective real curve such that $\bar{X}(\mathbb{R})$ has $\nu>0$ components. If $X$ is obtained by removing a real point from $\bar{X}$ then $X$ has $\nu$ components and $\lambda=\nu-1$ loops. Now we use lemma 7.1 to deduce the result from theorem 7.2. The extension to $K_{0}$ is just a rephrasing of Weichhold's theorem 1.1 .

The idea of the proof is to compute with the spectral sequence (6.2.0). For simplicity, we shall write $H^{n i}$ for $H_{M}^{n}(X, \mathbb{Z} / 2(i))$, which is the term $E_{2}^{n-i,-i}(\mathbb{Z} / 2)$. Here is a very useful trick.

Lemma 7.4. Fix $p<0$ and set $i=2-p$. Suppose that the $d_{2}$-differential $E_{2}^{p, p-2}=$ $H^{2, i} \rightarrow H^{5, i+1}$ has rank $r$. Then:

(1) The $d_{2}$-differential $E_{2}^{p, p-1}=H^{1, i-1} \rightarrow H^{4, i}$ has rank $\geq r$;

(2) The $d_{2}$-differential $E_{2}^{p, p-2-j}=H^{2+j, i+j} \rightarrow H^{5+j, i+j+1}$ has rank $r$ for all $j \geq 0$. 
Proof. The element $[-1] \in E_{2}^{0,-1}(\mathbb{Z})=H^{0}\left(X, \mathbb{G}_{m}\right)$ is a permanent cycle. By theorem 6.2, multiplication by this element commutes with the differentials in the spectral sequence $E_{2}^{* *}(\mathbb{Z} / 2)$. Hence we have a commutative diagram

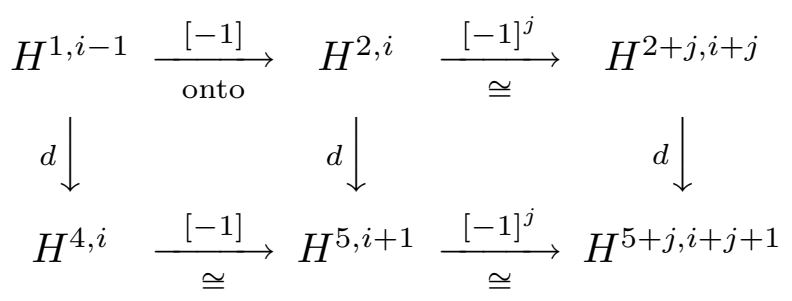

The horizontal maps are obtained by multiplication by $[-1]$; by 1.5 , they are all isomorphisms except for the upper left one, which is onto. The result follows.

Now every term in the spectral sequence (6.2.0) is a group of exponent two. We shall find it useful to abbreviate $(\mathbb{Z} / 2)^{n}$ with the integer " $n$," as in " $\nu$ " and " $\lambda$." The terms in the fourth quadrant of (6.2.0) are given by 1.4 , while the terms in the column $p=1$ come from 5.6. The left side of figure (7.5) gives the first few columns of the spectral sequence at $E_{2}$; every set of four columns to the left of this (from $p=-4 k$ to $-4 k-3$ ) is the same as the set depicted from $p=0$ to $p=-3$, shifted left 4 and down 4 .

$$
\begin{array}{cccccccccc}
\underline{-3} & -2 & -1 & \frac{p=0}{\mathbb{Z} / 2} & \frac{+1}{0} & \underline{-3} & \underline{-2} & \frac{-1}{11} & \frac{p=0}{\mathbb{Z} / 2} & \frac{+1}{0} \\
& & \mathbb{Z} / 2 & H^{11} & \lambda & & & \mathbb{Z} / 2 & H^{11} & \lambda \\
\mathbb{Z} / 2 & H^{13} & H^{12} & \nu+\lambda & \lambda & & 0 & \bar{H}^{12} & \nu+\lambda & \lambda \\
H^{14} & \nu+\lambda & \nu+\lambda & \nu+\lambda & \lambda & 0 & \tilde{H}^{13} & \nu & \nu+\lambda-1 & 0 \\
\nu+\lambda & \nu+\lambda & \nu+\lambda & \nu+\lambda & \lambda & g+r_{2} & 0 & \nu-1 & 0 & 0 \\
\nu+\lambda & \nu+\lambda & \nu+\lambda & \nu+\lambda & \lambda & \lambda & 0 & 0 & 0 & 0 \\
\multicolumn{2}{|c}{E_{2} \text { terms for } K_{*}(X, \mathbb{Z} / 2)} & \multicolumn{5}{c}{E_{3} \text { terms for } K_{*}(X, \mathbb{Z} / 2)} &
\end{array}
$$

Claim 7.6. We claim that the $E_{3}$ terms of the spectral sequence vanish for $p-q \geq 4$, and are given by the right side of (7.5), where the notation is as follows. The symbol $\bar{H}^{12}$ denotes the kernel of the surjection $H_{\mathrm{et}}^{1}(X, \mathbb{Z} / 2) \rightarrow(\mathbb{Z} / 2)^{\lambda}$, which has dimension $g+r_{1}+r_{2}$. The symbol $\tilde{H}^{13}$ denotes the kernel of the surjection $H_{\text {et }}^{1}(X, \mathbb{Z} / 2) \rightarrow(\mathbb{Z} / 2)^{\nu+\lambda}$ of lemma 1.5, a vector space of dimension $g+r_{2}-\lambda$.

Assuming this claim, we can now prove theorem 7.2. We will then devote the rest of this section to the column-by-column proof of our claim 7.6; the key results are 7.7.1 $(p=1), 7.9$ ( $p \leq 0$ even) and 7.10 ( $p<0$ odd $)$.

Proof of theorem 7.2. By inspection, there are no further differentials, and we may read off the associated graded groups for the filtration on $K_{*}(X, \mathbb{Z} / 2)$. We will focus on $K_{n}(X)$ for $2 \leq n \leq 9$, since the cases $n+8 k$ are entirely similar.

Clearly $K_{6}(X, \mathbb{Z} / 2)=0$, which implies that $K_{5}(X)$ is torsionfree and that $K_{6}(X)$ is divisible. By theorem 3.3 , the $(\mathbb{Q} / \mathbb{Z})^{g+r_{2}}$ in $K_{6}(X)$ accounts for all of $K_{7}(X, \mathbb{Z} / 2) \cong$ $(\mathbb{Z} / 2)^{g+r_{2}}$. Thus $K_{7}(X)$ is divisible. After accounting for the torsion subgroup $\mathbb{Q} / \mathbb{Z}$ 
of $K_{7}(X)$, the extension $K_{8}(X, \mathbb{Z} / 2)$ of $\mathbb{Z} / 2$ by $(\mathbb{Z} / 2)^{\lambda}$ yields $K_{8}(X) / 2 \cong(\mathbb{Z} / 2)^{\lambda}$. By theorem 3.3, this yields $K_{8}(X)_{\text {tors }} \cong(\mathbb{Q} / \mathbb{Z})^{g+r_{1}+r_{2}-1} \oplus(\mathbb{Z} / 2)^{\lambda}$.

From the extension $0 \rightarrow(\mathbb{Z} / 2)^{\lambda} \rightarrow K_{9}(X, \mathbb{Z} / 2) \rightarrow H^{15} \rightarrow 0$ and the knowledge that the dimension $g+r+\lambda-1$ of ${ }_{2}\left(K_{8} X\right)$ is one less than the dimension of $H^{15}$, it follows that $K_{9}(X) / 2 \cong(\mathbb{Z} / 2)^{1+\lambda}$. From 3.3 , the torsion subgroup of $K_{9}(X)$ has exponent 2 , so $K_{9}(X)_{\text {tors }} \cong K_{9}(X) / 2 \cong(\mathbb{Z} / 2)^{1+\lambda}$.

Now we move in the other direction. Since $K_{5}(X)$ is uniquely divisible, $K_{5}(X, \mathbb{Z} / 2) \cong$ ${ }_{2}\left(K_{4} X\right)$; by $(7.5), \operatorname{dim} K_{5}(X, \mathbb{Z} / 2)$ is $\left(g+r_{2}-\lambda\right)+(\nu-1)=g+r_{1}+r_{2}-1$, which exactly matches the dimension of ${ }_{2}\left(D_{4}\right)$. Hence $K_{4}(X)$ is divisible. But then the exponent 2 subgroup of $K_{3}(X)$ equals $K_{4}(X ; \mathbb{Z} / 2)$, a group which is $(\mathbb{Z} / 2)^{\nu}$ by $(7.5)$. By theorem 3.3 again, this yields $K_{3}(X)_{\text {tors }} \cong \mathbb{Q} / \mathbb{Z} \oplus(\mathbb{Z} / 2)^{\nu-1}$, agreeing with the computation in 4.9 .

To compute $K_{2}(X)_{\text {tors }}$, we turn to the extension

$$
0 \rightarrow(\mathbb{Z} / 2)^{\nu+\lambda-1} \rightarrow K_{3}(X, \mathbb{Z} / 2) \rightarrow \bar{H}^{12} \rightarrow 0
$$

From the computation of $K_{3}(X)_{\text {tors }}$ it follows that $K_{3}(X) / 2 \cong(\mathbb{Z} / 2)^{\nu-1}$, and hence ${ }_{2}\left(K_{2} X\right)$ has dimension $\operatorname{dim} \bar{H}^{12}+\lambda=g+r_{2}+\nu\left(\right.$ as $\left.\nu=r_{1}+\lambda\right)$. By 3.3 we have $K_{2}(X)=$ $D_{2} \oplus E_{2}$ with $\left(D_{2}\right)_{\text {tors }} \cong(\mathbb{Q} / \mathbb{Z})^{g+r_{2}}$. A dimension counts shows that the elementary abelian 2-group must be $E_{2} \cong(\mathbb{Z} / 2)^{\nu}$. Thus $K_{2}(X)_{\text {tors }} \cong(\mathbb{Q} / \mathbb{Z})^{g+r_{2}} \oplus(\mathbb{Z} / 2)^{\nu}$.

Continuing in this direction, we come to $K_{2}(X, \mathbb{Z} / 2)$; this is a group of cardinality $2^{1+\nu+\lambda}$ containing $K_{2}(\mathbb{R}, \mathbb{Z} / 2) \cong \mathbb{Z} / 4$. Since $K_{2}(X) / 2$ has dimension $\nu$, a count shows that ${ }_{2}\left(K_{1} X\right)$ has dimension $1+\lambda$. (Cf. theorem 4.1 .)

We have seen that the groups $K_{n}(X)_{\text {tors }}$ are determined for $2 \leq n \leq 9$ by (7.5) and theorem 3.3. By periodicity of (7.5), we may add a multiple of 8 to all subscripts and use the same argument to determine the groups $K_{n}(X)_{\text {tors }}$ for all $n$.

We now turn to the proof of the claim 7.6. Recall that $N H^{n i}$ is the kernel of $H^{n i}(X) \rightarrow H^{n i}(F)$. By 1.4 and 5.4, NH$H^{n i} \cong(\mathbb{Z} / 2)^{\lambda}$ whenever $2 \leq n \leq i+1$.

Lemma 7.7. If $i>0$ and $i \cong 3,4(\bmod 4)$, the differential $d_{X}: H^{2, i} \rightarrow H^{5, i+1}$ induces an isomorphism $N H^{2, i} \cong N H^{5, i+1}$.

Proof. The differential $H^{0, i-1}(\mathbb{R}) \stackrel{d}{\rightarrow} H^{3, i}(\mathbb{R})$ is an isomorphism in the spectral sequence for $\operatorname{Spec}(\mathbb{R})$ by $[R W, 5.3]$. By naturality and theorem 6.2, we can multiply by $E_{2}^{1,-1}(\mathbb{Z})=H_{M}^{2}(X, \mathbb{Z}(1))=\operatorname{Pic}(X)$; because all differentials vanish on $\operatorname{Pic}(X)$, this multiplication commutes with the differentials in the spectral sequence for $X$. This yields the commutative diagram:

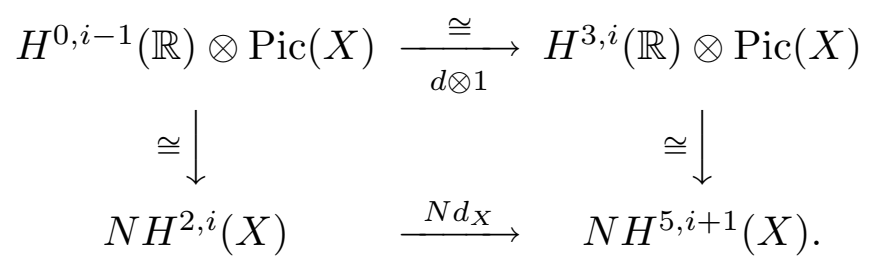

Now $\operatorname{Pic}(X) / 2 \cong N H_{\text {et }}^{2}(X, \mathbb{Z} / 2)$ and $H^{p i}(\mathbb{R}) \cong H_{\text {et }}^{p}(\mathbb{R}, \mathbb{Z} / 2) \cong \mathbb{Z} / 2$ on $[-1]^{p}$ for $0 \leq p \leq$ $i$. Hence the vertical maps are isomorphisms by 1.6, and this implies that the bottom arrow $N d_{X}$ in (7.7.0) is also an isomorphism. 
In particular, when $i=3$ the differential from $E_{2}^{-1,-3}(\mathbb{Z} / 2)=H^{23}$ to $E_{2}^{1,-4}=H_{M}^{54} \cong$ $N H^{54} \cong(\mathbb{Z} / 2)^{\lambda}$ is onto. Now $E_{2}^{1 q} \cong(\mathbb{Z} / 2)^{\lambda}$ for all $q<0$. Combining these facts with lemma 7.4 for $p=-1$, we obtain the following corollary.

Corollary 7.7.1. The $d_{2}$-differentials $E_{2}^{-1, q} \rightarrow E_{2}^{1, q-1} \cong(\mathbb{Z} / 2)^{\lambda}$ are onto for all $q \leq-2$.

To describe the differentials for $p<-1$ we resort to a comparison with the no loops case, which was described in (6.7).

Lemma 7.8. Let $U$ be the subscheme of $X$ obtained by removing one point from each of the $\lambda$ loops on $X$. Then $H_{\mathrm{et}}^{n}(X, \mathbb{Z} / 2) \rightarrow H_{\mathrm{et}}^{n}(U, \mathbb{Z} / 2)$ is onto for all $n$, and is an isomorphism for $n \leq 1$.

Proof. Note that $\lambda(U)=0$ but $\nu(U)=\nu(X)$ and $r_{2}(U)=r_{2}(X)$. The lemma now follows from the localization sequence

$$
0 \rightarrow H_{\mathrm{et}}^{1}(X, \mathbb{Z} / 2) \rightarrow H_{\mathrm{et}}^{1}(U, \mathbb{Z} / 2) \rightarrow(\mathbb{Z} / 2)^{\lambda} \rightarrow H_{\mathrm{et}}^{2}(X, \mathbb{Z} / 2) \rightarrow H_{\mathrm{et}}^{2}(U, \mathbb{Z} / 2) \cdots
$$

and a dimension count from 1.4: $\operatorname{dim} H_{\mathrm{et}}^{1}(X, \mathbb{Z} / 2)=\operatorname{dim} H_{\mathrm{et}}^{1}(U, \mathbb{Z} / 2)=g+r_{2}+\nu$ and $\operatorname{dim} H_{\mathrm{et}}^{n}(X, \mathbb{Z} / 2)=\nu+\lambda=\operatorname{dim} H_{\mathrm{et}}^{n}(U, \mathbb{Z} / 2)+\lambda$ for all $n \geq 2$.

There is a morphism of spectral sequences which on the $E_{2}$ level are the maps $H_{M}^{*}(X, \mathbb{Z} / 2(i)) \rightarrow H_{M}^{*}(U, \mathbb{Z} / 2(i))$.

Corollary 7.8.1. Suppose that $p<0$. (a) When either $p \equiv 0$ or $p \equiv-1(\bmod 4)$, the differential $E_{2}^{p p} \rightarrow E_{2}^{p+2, p-1}$ is zero. Hence $E_{3}^{p p}=\mathbb{Z} / 2$.

(b) When either $p \equiv-2$ or $p \equiv-3(\bmod 4)$, the differential $E_{2}^{p p} \rightarrow E_{2}^{p+2, p-1}$ is an injection. Hence $E_{3}^{p p}=0$.

Proof. This follows from chasing the following commutative diagram with $i=-p$ :

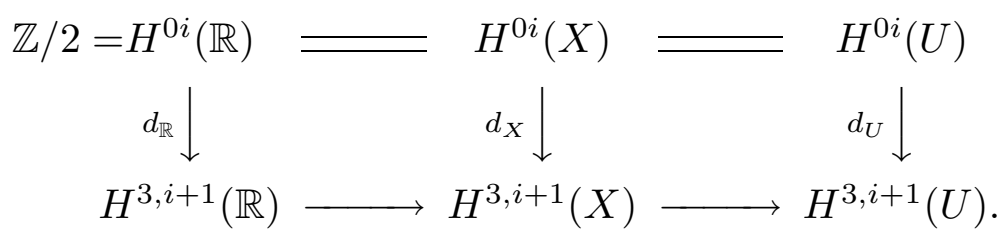

The maps in the bottom row are induced by the closed embeddings Spec $\mathbb{R} \hookrightarrow X$ (for each of the $\lambda$ points of $X-U)$ and by the open inclusion $U \subset X$.

When $p \equiv 0$ or $p \equiv-1(\bmod 4)$, the left vertical $d_{\mathbb{R}}$ is zero by $[\mathrm{RW}, 5.3]$, so $d_{X}=0$. When $p \equiv-2$ or $p \equiv-3(\bmod 4)$, we saw in $(6.7)$ that the right vertical $d_{U}$ is an injection, so $d_{X}$ must also be an injection.

Corollary 7.8.2. If $i \geq 3$, the $d_{2}$-differential $H^{2, i}(X) \stackrel{d_{X}}{\longrightarrow} H^{5, i+1}(X)$ is:

(1) an isomorphism if $i \equiv 0(\bmod 4)$;

(2) of rank $\nu$ if $i \equiv 1(\bmod 4)$;

(3) of $\operatorname{rank} \lambda$ if $i \equiv 3(\bmod 4)$.

We will see in 7.9 that $d_{X}=0$ in the missing case $i \equiv 2(\bmod 4)$. 
Proof. Using lemma 7.8 and 1.4, the rows are exact in the commutative diagram:

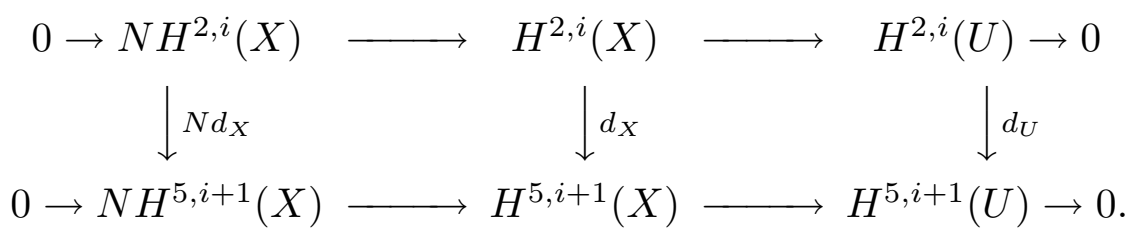

By lemma 7.7, the left vertical map $N d_{X}$ is an isomorphism when $i \equiv 3,4(\bmod 4)$, and zero if $i \equiv 1,2(\bmod 4)$. By $(6.7)$, the right vertical $d_{U}$ is an isomorphism when $i \equiv 0,1(\bmod 4)$, and zero if $i \equiv 2,3(\bmod 4)$. When $i \equiv 0(\bmod 4), \operatorname{both} N d_{X}$ and $d_{U}$ are isomorphisms; hence $d_{X}$ is an isomorphism. When $i \equiv 1(\bmod 4)$, the map $N d_{X}$ is zero and hence $d_{X}$ has rank $\nu$. When $i \equiv 3(\bmod 4)$, the map $d_{U}$ is zero and hence $d_{X}$ has rank $\lambda$.

Since we are interested in the columns of (7.5), it is convenient to index things by $p=2-i$. We first handle the even columns.

Lemma 7.9. When $p \equiv 2(\bmod 4)$ and $p$ is negative,

(1) $E_{3}^{p q}=0$ for all $q \neq p-1$;

(2) $E_{3}^{p, p-1}$ is the kernel $\tilde{H}^{1,1-p}$ of the surjection $H^{1,1-p} \stackrel{d}{\rightarrow} H^{4,2-p} \cong(\mathbb{Z} / 2)^{\nu+\lambda}$. Hence $E_{3}^{p, p-1} \cong(\mathbb{Z} / 2)^{g+r_{2}-\lambda}$.

When $p \equiv 0(\bmod 4)$ and $p \leq 0$,

(1) $E_{3}^{p q}=0$ for all $q \leq p-4$;

(2) $E_{3}^{p, p-3}=H^{3,3-p} /(\mathbb{Z} / 2)$ has rank $\nu+\lambda-1$.

(3) $E_{3}^{p p}=\mathbb{Z} / 2, E_{3}^{p, p-1}=E_{2}^{p, p-1}=H^{1,1-p}$ and $E_{3}^{p, p-2}=E_{2}^{p, p-2}=(\mathbb{Z} / 2)^{\nu+\lambda}$.

Proof. First assume that $p \equiv 2(\bmod 4)$ and set $i=2-p>0$. We saw in 7.8.2 that the differential $E_{2}^{p, p-2}=H^{2, i} \rightarrow H^{5, i+1}$ is an isomorphism, so it has full rank $\nu+\lambda$. By 7.4, $E_{2}^{p q} \rightarrow E_{2}^{p+2, q-1} \cong(\mathbb{Z} / 2)^{\nu+\lambda}$ also has full rank $\nu+\lambda$ (i.e., is onto) for all $q<p$. Hence $E_{3}^{p+2, q-1}=0$ for $q \leq p-1$. For $q=p-1$ we have $E_{2}^{p q}=H^{1,1-p}$ so $E_{3}^{p, p-1}=\tilde{H}^{1,1-p}$. For $q \leq p-2$ we have $E_{2}^{p q} \cong(\mathbb{Z} / 2)^{\nu+\lambda}$ so $E_{3}^{p q}=0$. Finally, the case $q=p$ is handled in 7.8.1.

When $p \equiv 0(\bmod 4)$ we argue as follows. We have already seen that $E_{3}^{p q}=0$ if $q \leq p-4$ in the last paragraph. If $p=0$ and $q>-4$, the description of $E_{3}^{0 q}$ is immediate from (7.5) and 7.8.1(b). For $p<0$ we have chain complexes:

$$
\begin{gathered}
0 \rightarrow E_{2}^{p p} \stackrel{d}{\rightarrow} E_{2}^{p+2, p-1} \stackrel{d \cong}{\longrightarrow} E_{2}^{p+4, p-2} \\
0 \rightarrow E_{2}^{p, p-1} \stackrel{d}{\rightarrow} E_{2}^{p+2, p-2} \stackrel{d \cong}{\longrightarrow} E_{2}^{p+4, p-3} \\
0 \rightarrow E_{2}^{p, p-2} \stackrel{d}{\rightarrow} E_{2}^{p+2, p-3} \stackrel{d \cong}{\longrightarrow} E_{2}^{p+4, p-4}
\end{gathered}
$$

We have seen that the second differential is an isomorphism, so the first differentials must be zero. Thus $E_{3}^{p q}=E_{2}^{p q}$ for $q=p, p-1, p-2$. This proves (3). Finally, we use the chain complex

$$
0 \rightarrow E_{2}^{p-2, p-2} \underset{\text { into }}{\stackrel{d}{\longrightarrow}} E_{2}^{p, p-3} \stackrel{d}{\rightarrow} E_{2}^{p+2, p-4} \stackrel{d \cong}{\longrightarrow} E_{2}^{p+4, p-5} .
$$


Again, we have seen that the third differential is an isomorphism, so the second differential must be zero. We have also shown in 7.8.1 that the first differential is an injection. Since $E_{2}^{p, p-3} \cong(\mathbb{Z} / 2)^{\nu+\lambda}$, we get $E_{3}^{p, p-3} \cong(\mathbb{Z} / 2)^{\nu+\lambda-1}$, as claimed in $(2)$.

Lemma 7.10. When $p \equiv-3(\bmod 4)$ and $p$ is negative, then $E_{3}^{p q}=0$ for all $q \leq p-4$. Also $E_{3}^{p p}=0$ by 7.8.1, $E_{3}^{p, p-1} \cong(\mathbb{Z} / 2)^{g+r_{2}}$, and $E_{3}^{p, p-2} \cong E_{3}^{p, p-3} \cong(\mathbb{Z} / 2)^{\lambda}$.

When $p \equiv-1(\bmod 4)$ and $p$ is negative, then $E_{3}^{p q}=0$ for all $q \leq p-4$. Also $E_{3}^{p p} \cong \mathbb{Z} / 2, E_{3}^{p, p-1} \cong \bar{H}^{1,1-p}, E_{3}^{p, p-2} \cong(\mathbb{Z} / 2)^{\nu}$ and $E_{3}^{p, p-3} \cong(\mathbb{Z} / 2)^{\nu-1}$.

Proof. When $p=4 k-1$ and $q \leq p-4$ the group $E_{2}^{p q} \cong(\mathbb{Z} / 2)^{\nu+\lambda}$ fits into a chain complex

$$
E_{2}^{p-2, q+1} \stackrel{\nu}{\longrightarrow} E_{2}^{p q} \stackrel{\lambda}{\longrightarrow} E_{2}^{p+2, q-1} .
$$

By 7.7.1, 7.8.2 and 7.4, the map labeled ' $\lambda$ ' has rank $\lambda$ and the map labeled ' $\nu$ ' has rank $\nu$; the case $q=p-4$ requires a dimension count in addition to 7.4(1). Similarly, when $p=4 k-3$ and $q \leq p-4$ the group $E_{2}^{p q} \cong(\mathbb{Z} / 2)^{\nu+\lambda}$ fits into a chain complex

$$
E_{2}^{p-2, q+1} \stackrel{\lambda}{\rightarrow} E_{2}^{p q} \stackrel{\nu}{\rightarrow} E_{2}^{p+2, q-1} .
$$

Again by 7.7.1, 7.8.2 and 7.4 (and a dimension count), the map labeled ' $\nu$ ' has rank $\nu$ and the map labeled ' $\lambda$ ' has rank $\lambda$. It follows that $E_{3}^{p q}=0$ in both of these cases.

We need more ad hoc arguments when $q \geq p-3$. The case $q=p$ is given by 7.8.1. When $p=-1$, we know from 7.7.1 that $E_{2}^{-1, q} \rightarrow E_{2}^{1, q-1}=(\mathbb{Z} / 2)^{\lambda}$ is onto for $q \leq-2$. The calculations of $E_{3}^{-1, q}$ are immediate for $q=-2,-3$, and for $q=-4$ using 7.8 .1 with $p=-3$.

Now suppose that $p<-1$ and $p$ is odd. When $q=p-2$, we know from 7.8.2 that there is a chain complex like (7.10.1) or (7.10.2), except that the leftmost term is zero; this yields the given descriptions of $E_{3}^{p, p-2}$. When $q=p-1$ and $p \equiv-1$ the extended version of (7.10.1) is:

$$
0 \rightarrow E_{2}^{p, p-1} \stackrel{d}{\rightarrow} E_{2}^{p+2, p-2} \stackrel{\nu}{\rightarrow} E_{2}^{p+4, p-3} .
$$

The map $d$ has rank at least $\lambda$, and the map ' $\nu$ ' has rank exactly $\nu$ by 7.8 .2 and 7.4. Since $E_{2}^{p+2, q-1} \cong(\mathbb{Z} / 2)^{\nu+\lambda}$ it follows that $d$ has rank exactly $\lambda$, giving $E_{3}^{p, p-1}=\bar{H}^{1,1-p}$. The argument for $q=p-1$ and $p \equiv-3$ is identical, with $\nu$ and $\lambda$ interchanged.

Finally, to compute $E_{3}^{p, p-3}$ we consider the chain complex:

$$
E_{2}^{p-2, p-2} \stackrel{d}{\rightarrow} E_{2}^{p, p-3} \stackrel{d}{\rightarrow} E_{2}^{p+2, p-4} .
$$

If $p \equiv-1(\bmod 4)$, we know from 7.8.1 that the left differential $d$ is an injection, and from 7.8.2 and 7.4 that the right differential $d$ has rank $\lambda$. Since $E_{2}^{p, p-3}$ has rank $\nu+\lambda$ and $E_{2}^{p-2, p-2}=\mathbb{Z} / 2$, we have $E_{3}^{p, p-3} \cong(\mathbb{Z} / 2)^{\nu-1}$.

If $p \equiv-3(\bmod 4)$, we know from 7.8 .1 that the left differential $d$ is zero, and from 7.8.2 and 7.4 that the right differential $d$ has rank $\nu$. Since $E_{2}^{p, p-3}$ has rank $\nu+\lambda$ we have $E_{3}^{p, p-3} \cong(\mathbb{Z} / 2)^{\lambda}$.

This completes the verification of claim 7.6, and hence the proofs of both theorem 7.2 and the main theorem 0.1 . 


\section{REFERENCES}

[A] M. Atiyah, K-theory and reality, Quart. J. Math. 17 (1966), 367-86, reprinted in the book $K$-theory, Benjamin, 1967.

[AD] R. Alperin and R. K. Dennis, $K_{2}$ of Quaternion algebras, J. Alg. 56 (1979), 262-273.

[Bl] S. Bloch, Algebraic cycles and higher K-theory, Adv. Math. 61 (1986), 267-304.

[Cox] D. Cox, The étale homotopy type of varieties over R, Proc. AMS 76 (1979), 17-22.

[CP] C. Ciliberto and C. Pedrini, Real abelian varieties and real algebraic curves, Lectures in Real Geometry, W. De Gruyter, 1997, pp. 168-256.

[CT-P] J.-L. Colliot-Thelene and R. Parimala, Real components of algebraic varieties and étale cohomology, Inv. Math 101 (1990), 81-99.

[CT-S] J.-L. Colliot-Thelene and C. Scheiderer, Zero-cycles and cohomology on real algebraic varieties, Topology 35 (1996), 533-559.

[DK] F. Demeyer and M. Knus, The Brauer group of a real curve, Proc. AMS 57 (1976), 227-232.

[Fr] E. Friedlander, Motivic complexes of Suslin and Voevodsky, Exp. 833 of Séminaire Bourbaki, 1996/97, Astérisque 245 (1997), 355-378.

[FS] E. Friedlander and A. Suslin, The spectral sequence relating algebraic $K$-theory to motivic cohomology, preliminary draft (1999), www.math.uiuc.edu/K-theory/0360.

[FV] E. Friedlander and V. Voevodsky, Bivariant cycle cohomology, Preprint (1995), Cycles, transfers and motivic homology theories, Princeton University Press, 2000, pp. 138-187.

[Ger] S. Gersten, Higher K-theory of rings, Lecture Notes in Math., vol. 341, Springer Verlag, 1973.

[K] M. Karoubi, Algèbres de Clifford et K-théorie, Ann. Sci. École Norm. Sup. 1 (1968), 161-270.

[KW] M. Karoubi and C. Weibel, Algebraic K-theory and KR-theory of Real varieties, In preparation.

[L] M. Levine, K-theory and motivic cohomology of schemes, unpublished preprint (1999), posted at www.math.uiuc.edu/K-theory/0336.

[Mi] J. Milne, Étale Cohomology, Princeton Univ. Press, 1980.

[MS] A. Merkurjev and A. Suslin, The group $K_{3}$ for a field, Izv. Akad. Nauk SSSR Ser. Mat. 54 (1990), 522-545 (Russian); transl., Math. USSR Izvestiya 36 (1991), 541-565.

[MS1] , Norm residue homomorphism of degree three, Izv. Akad. Nauk SSSR Ser. Mat. 54 (1990), 339-356. (Russian)

[O] S. Oka, Unpublished letter.

[PW1] C. Pedrini and C. Weibel, Invariants of real curves, Rend. Seminario Mat. Univ. Torino 49 (1991), 139-173.

[PW2] C. Pedrini and C. Weibel, The higher K-theory of a Complex Surface, Compositio Math. (submitted) (1999).

[PW3] C. Pedrini and C. Weibel, Divisibility in the Chow group of zero-cycles on a singular surface, Astérisque 226 (1994), 371-409.

[RbW] L. Roberts and C. Weibel, $K_{2}$ and $K_{3}$ of the circle, J. Pure Appl. Alg. 23 (1982), 67-95.

[RW] J. Rognes and C. Weibel, Two-primary algebraic $K$-theory of rings of integers in number fields, Jour. AMS 13 (2000), 1-54.

[Sch] C. Scheiderer, Real and Étale Cohomology, Lecture Notes in Math. 1588, Springer-Verlag, 1994.

[Sil] R. Silhol, Real abelian varieties and the theory of Comessatti, Math. Z. 181 (1982), 345-364.

[Su1] A. Suslin, Algebraic K-theory of fields, Proc. 1986 I.C.M. Berkeley, 1987, pp. 222-244.

[Su2] A. Suslin, Torsion in $K_{2}$ of fields, K-theory 1 (1987), 5-29.

[Su3] A. Suslin, Higher Chow groups and étale cohomology, Preprint (1994), Cycles, transfers and motivic homology theories, Princeton Univ. Press, 2000, pp. 239-254.

[SuM] A. Suslin, Algebraic K-theory and motivic cohomology, Proc. 1994 I.C.M. Zürich, Birkhäuser, 1995, pp. 342-351.

[SV] A. Suslin and V. Voevodsky, Bloch-Kato conjecture and motivic cohomology with finite coefficients, The Arithmetic and Geometry of Algebraic Cycles, NATO Science Series C, vol. 548, Kluwer, 2000, pp. 117-189.

[V] V. Voevodsky, The Milnor Conjecture, preprint (1996), www.math.uiuc.edu/K-theory/0170. 
[W] C. Weibel, Products in Chow groups and Motivic cohomology, Proc. Symp. Pure Math., vol. 67, 1999, pp. 305-315..

[Wp] C. Weibel, Mayer-Vietoris sequences and $\bmod p$ K-theory, Lecture Notes in Math., vol. 966 , Springer-Verlag, 1982, pp. 390-407.

[Whd] G. Weichold, Ueber symmetrische Riemann'sche Fläschen und die Periodicitätsmoduln der zugehörigen Abel'schen Normalintegrale erster Gattung (sic), Zeitschrift f. Math. u. Phys. 28 (1883), 321-351.

[Witt] E. Witt, Zerlegung reeller algebraischer Funktionen in Quadrate. Schiefkörper über reellem Functionenkörper, J. Reine Angew. Math. 171 (1934), 4-11. 